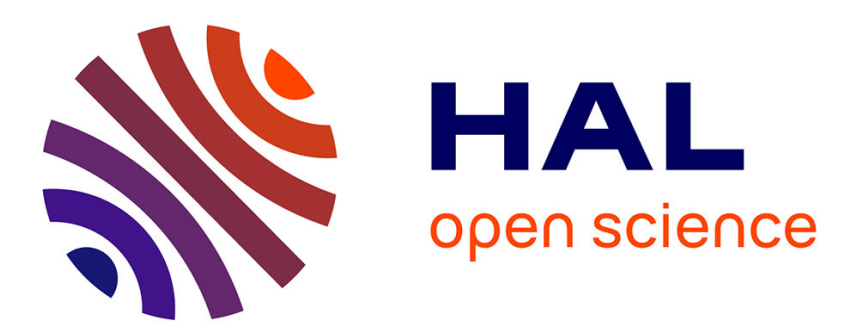

\title{
Valeurs affiliées et comportements d'offre: analyse théorique et application aux exportations de céréales européennes
}

\author{
Jean-Marc Bourgeon, Yves Le Roux
}

\section{To cite this version:}

Jean-Marc Bourgeon, Yves Le Roux. Valeurs affiliées et comportements d'offre: analyse théorique et application aux exportations de céréales européennes. [Rapport de recherche] Inconnu. 1997, 33 p. hal-02327561

\section{HAL Id: hal-02327561 \\ https://hal.science/hal-02327561}

Submitted on 22 Oct 2019

HAL is a multi-disciplinary open access archive for the deposit and dissemination of scientific research documents, whether they are published or not. The documents may come from teaching and research institutions in France or abroad, or from public or private research centers.
L'archive ouverte pluridisciplinaire HAL, est destinée au dépôt et à la diffusion de documents scientifiques de niveau recherche, publiés ou non, émanant des établissements d'enseignement et de recherche français ou étrangers, des laboratoires publics ou privés.

\section{(1) (1) $\$$}

Distributed under a Creative Commons Attribution - NonCommercial - NoDerivatives $\mid 4.0$ 


\title{
Valeurs affiliées et comportements d'offre: analyse théorique et application aux exportations de céréales européennes*
}

\author{
Jean-Marc Bourgeon ${ }^{\dagger} \quad$ Yves Le Roux ${ }^{\ddagger}$
}

16 octobre 1997

\begin{abstract}
Résumé
Grâce à une spécification d'une loi multivariée présentant de faibles corrélations due à Gouriéroux et Jouneaux (1994), nous modélisons le comportement d'offre lors d'une enchère sous plis cachetés au premier prix sous les hypothèses de valeurs privées et de valeur commune. Nous généralisons ensuite la procédure d'estimation développée dans Bourgeon et Le Roux (1995) dans le cas de la valeur commune et nous l'appliquons aux exportations européennes de céréales.
\end{abstract}

\section{Introduction}

L'Union Européenne (UE) détient un rôle important sur le marché international des céréales: la part de ses exportations dans le total du commerce mondial se maintient entre 15 et $20 \%$ depuis le début des années 1990 . Ces exportations peuvent avoir deux origines: le marché intérieur européen sur lequel peuvent intervenir les compagnies de négoce, ou les stocks communautaires, que les organismes chargés d'intervenir sur le marché constituent du fait de l'engagement de soutien du prix de l'UE auprès des agriculteurs. La vente de ces stocks s'effectue au moyen d'adjudications hebdomadaires, après appels d'offres auprès des compagnies de négoce.

*Cette recherche a bénéficié d'un financement de l'INRA dans le cadre de l'AIP "Régulation des marchés".

†THEMA - Université Paris-X, Nanterre.

${ }^{\dagger}$ INRA - Rennes 
Deux paradigmes opposés, issus de la littérature sur la théorie des enchères, peuvent être retenus pour caractériser le comportement des compagnies de négoce lors de ces adjudications de stocks d'intervention. Ces paradigmes diffèrent par les évaluations individuelles du bien mis en vente que forment les agents, suivant qu'il s'agit de "valeurs privées" ou d'une "valeur commune". Dans le cadre du paradigme de "valeurs privees", chaque agent attache une valeur particulière au bien vendu de façon certaine et avant l'enchère. On suppose généralement que ces valeurs sont différentes et indépendantes entre les agents. L'hypothèse de valeurs privées peut se vérifier dans le cas d'une segmentation du marché mondial, et pourrait être expliquée par le fait que certains opérateurs détiennent un pouvoir de marché spécifique. Par contre, dans le cadre du paradigme de "valeur commune", tous les agents attachent au bien vendu une même valeur ex post, mais cette valeur leur est inconnue ex ante. Avant le déroulement de l'enchère, les agents ont simplement des estimations de cette valeur, fonctions de l'information dont ils disposent, ou "signal".

L'objet de cet article est de spécifier et d'estimer le comportement des opérateurs lors des adjudications des stocks d'intervention de l'UE, sous l'hypothèse que ces opérateurs forment des estimations (faiblement) corrélées des lots soumis à adjudication. Dans un précédent article (voir Bourgeon et Le Roux, 1995) nous proposons une méthode d'estimation permettant de déterminer lequel de ces deux cadres d'analyse est le plus approprié aux adjudications européennes, sous l'hypothèse que les valeurs ou les signaux des offreurs sont des réalisations de variables aléatoires indépendantes, issues de lois normales ou log-normales. On peut en effet, sous ces hypothèses, et pour chaque paradigme, expliciter les moments du premier et du second ordre des offres gagnantes, ce qui permet de dégager quelques résultats. En retenant des hypothèses similaires dans chaque cadre, il apparaît que les formes réduites des équations à estimer sont les mêmes sous les deux paradigmes (que les lois latentes soient normales ou log-normales), de sorte qu'il n'est pas possible de trancher statistiquement sur la question du paradigme pertinent. ${ }^{1}$ Cependant, les spécifications retenues permettent une estimation simple des paramètres caractérisant les distributions sous les deux paradigmes. L'estimation statistique est en effet opérée en deux étapes, la première consistant en une régression linéaire (en niveau ou sur le logarithme des variables), la seconde utilisant les résultats de cette estimation pour déduire le paramètre de variance. Bien que l'on ne puisse pas trancher statistiquement, les résultats obtenus suggèrent toutefois qu'il est peu vraisemblable de retenir le paradigme des valeurs privées dans le cas du marché mondial des céréales.

\footnotetext{
${ }^{1}$ Sur cette question, voir Laffont et Vuong 1996.
} 
Nous proposons une généralisation de ce cadre d'analyse en levant l'hypothèse d'indépendance des distributions latentes. La prise en compte de telles corrélations permet de s'affranchir de l'hypothèse usuelle selon laquelle les valorisations du bien mis aux enchères (ou leurs anticipations) sont indépendantes parmi les opérateurs. Cette généralisation est rendue possible grâce à une famille particulière de lois multivariées présentant de faibles dépendances entre leurs composantes, stable par marginalisation et qui permet notamment une explicitation des statistiques d'ordre associées (voir Gouriéroux et Jouneau, 1994). Il est ainsi possible de spécifier les fonctions d'offre des opérateurs sous les deux paradigmes, ce qui conduit à analyser l'effet des corrélations sur ces comportements. On obtient en particulier des évolutions différentes suivant le paradigme retenu: dans le cas de la valeur commune (restreint à un cadre d'hypothèses dû à Paarsch, 1992, qui permet l'obtention de fonctions d'offre linéaires), les offres avec corrélations sont toujours supérieures aux offres sous l'hypothèse d'indépendance, alors que dans le cas de valeurs privées les corrélations ont un effet ambigu sur les comportements, avec toutefois une offre plus faible que dans le cas d'indépendance sur la majeure partie du support des valeurs possibles. Dans le cas de valeurs privées en effet, comparativement à la situation d'indépendance, les corrélations induisent des offres plus faibles pour de faibles valeurs privées et plus fortes dans le cas de valeurs privées importantes, mais une valeur privée est forte ou faible relativement au nombre d'offreurs en concurrence, car elle doit être comparée non pas à l'espérance de la distribution de référence mais à un seuil qui croît rapidement en fonction du nombre de participants.

Il est par ailleurs possible avec la famille de distributions retenue de spécifier, sous le paradigme de la valeur commune, les espérances et les variances des deux premières offres. On peut par conséquent estimer les paramètres de la distribution par une approche en deux étapes similaire à celle développée dans le cas d'indépendance, et qui, elle aussi, ne nécessite qu'une simple régression linéaire (méthode des moindres carrés asymptotiques). Toutefois, comme le souligne l'application qui suit, la valeur du paramètre d'affiliation obtenue dans la deuxième étape est très sensible aux niveaux des coefficients estimés. C'est pourquoi nous effectuons aussi une estimation directe de l'ensemble des paramètres par les moindres carrés non-linéaires, en utilisant les moments théoriques non-centrés du deuxième ordre et les séries des carrés des variables endogènes. Les résultats obtenus par les deux méthodes montrent l'existence d'une forte corrélation entre les signaux dans le cas des exportations européennes de céréales.

La suite de l'article est organisée de la manière suivante: dans une seconde partie, nous spécifions les comportements d'offre des opérateurs sous les deux paradigmes. La troisième section est consacrée aux méthodes d'esti- 
mation, qui prennent en compte l'hypothèse de corrélation. Dans la quatrième section, sont présentés les résultats obtenus par application de ces méthodes à un échantillon d'adjudications de stocks d'intervention de blé tendre.

\section{La modélisation des enchères}

Nous allons présenter dans ce qui suit une modélisation très générale du comportement des offreurs lors d'une enchère sous plis cachetés au premier prix due à Milgrom et Weber (1982). Ces auteurs supposent que la valeur qu'un individu va finalement reconnaître à l'objet mis en vente dépendra non seulement de ses caractéristiques intrinsèques, inconnue des offreurs au moment de l'enchère, mais aussi de l'intérêt particulier que les autres participants et luimême attachent à cet objet. On notera $a_{i}$ la réalisation de la variable aléatoire $A_{i}$ du signal propre à l'individu $i$ que celui-ci perçoit de l'objet avant l'enchère. De la même manière on notera $v_{i}=v\left(c, a_{i}, a_{-i}\right)$ la valeur qu'il attribue à l'objet après l'enchère, connaissant l'ensemble des signaux des autres offreurs, $a_{-i}$, et l'ensemble des caractéristiques intrinsèques de l'objet, $c,{ }^{2}$ et par $V_{i}=v\left(C, a_{i}, A_{-i}\right)$ la variable aléatoire correspondant à cette valeur ex ante, lorsque l'individu $i$ ne connaît pas les évaluations des autres offreurs (qui sont représentées par le vecteur des $n-1$ variables aléatoires $A_{-i}$ ) ni les caractéristiques de l'objet (variable aléatoire $C$ ). L'hypothèse usuelle de symétrie des offreurs dans les modèles d'enchère s'étend à la fonction d'évaluation $v(\cdot)$, qui doit par conséquent être symétrique dans ces $n-1$ derniers arguments. Cette fonction permet au modèle d'englober deux cas extrêmes: le premier cas est celui des valeurs privées indépendantes, qui correspond à une valeur. de l'objet égale à l'évaluation des individus et qui est indépendante des évaluations des autres participants: $v_{i}=v\left(c, a_{i}, a_{-i}\right)=a_{i}$ pour tout $c$ et $a_{-i}$. L'autre cas correspond en revanche à une valeur de l'objet commune à tous les participants (par exemple sa valeur de revente): $v_{i}=v\left(c, a_{i}, a_{-i}\right)=c$. Dans ce dernier cas, les estimations des individus sont des réalisations de variables aléatoires conditionnelles à cette valeur commune. La généralisation proposée par Milgrom et Weber ne s'arrête cependant pas à l'introduction de cette fonction d'évaluation abstraite. Ils introduisent aussi la possibilité de corrélations entre les signaux et la valeur de l'objet, en supposant que le choix de la nature, le $(n+1)$-uplet $Z \equiv\left(C, A_{i}\right)_{i=1, \ldots, n}$, admet une distribution de probabilité invariante par permutation des signaux (hypothèse de symétrie)

\footnotetext{
${ }^{2}$ Milgrom et Weber considerent un vecteur de caractéristiques qui peuvent correspondre à autant de paramêtres qualitatifs, inconnus des offreurs aux moment de l'enchère. Nous retiendrons par la suite l'interprétation de valeur de revente de l'objet qui correspond au paradigme de valeur commune.
} 
et respectant l'hypothèse d'affiliation, c'est-à-dire (en notant $f$ la densité), :

$$
\forall z, z^{\prime}, f\left(z \vee z^{\prime}\right) f\left(z \wedge z^{\prime}\right) \geq f(z) f\left(z^{\prime}\right)
$$

où $\left(z \vee z^{\prime}\right)$ (resp. $\left(z \wedge z^{\prime}\right)$ ) désigne le vecteur des composantes maximales (respectivement minimales) des deux vecteurs $z$ et $z^{\prime}$. Cette hypothèse d'affiliation n'autorise en fait que des corrélations positives entre les variables aléatoires (elle est cependant plus générale qu'une simple hypothèse sur les corrélations). Par conséquent, si en participant à une enchère sous pli cacheté au premier prix, l'offreur $i$ n'apprend rien du déroulement du jeu sur la valeur de l'objet (car il ne peut pas observer le comportement des autres offreurs), son signal $a_{i}$ lui permet cependant d'associer des niveaux de probabilité à différentes valeurs des qualités intrinsèques de l'objet, ce qui doit être pris en compte dans son appréciation de la valeur de l'objet. Par ailleurs, le fait qu'à l'équilibre une offre $b_{i}$ soit gagnante lui apporte de l'information sur les signaux des autres joueurs. Nous allons développer tous ces points en détail. Un individu $i$ peut en adoptant la stratégie d'offre $b_{i}$ réaliser un profit qui s'exprime, étant données les stratégies $b_{-i}$ des autres offreurs, leurs signaux $a_{-i}$ et les qualités de l'objet $c$ :

$$
\Pi_{i}\left(a_{i}, b_{i}\right)=\left[v_{i}-b_{i}\right] 1_{\left\{\forall j \neq i, b_{j}<b_{i}\right\}}
$$

où $1_{\{\omega\}}$ est la fonction indicatrice égale à 1 si l'évènement $\omega$ est réalisé, 0 sinon.

$\mathrm{Ne}$ connaissant ni les caractéristiques intrinsèques de l'objet, ni les estimations des autres offreurs, il n'a qu'une estimation de la valeur de l'objet et par conséquent son profit espéré s'exprime, pour un profil de stratégies $B_{-i}$ quelconque:

$$
E \Pi_{i}\left(a_{i}, b_{i}\right)=E\left[\left(V_{i}-b_{i}\right) 1_{\left\{\forall j \neq i, B_{j}<b_{i}\right\}} \mid A_{i}=a_{i}\right]
$$

En supposant que les autres offreurs jouent leurs stratégies d'équilibre $B_{-i}^{*}=b^{*}\left(A_{-i}\right)$ et que la fonction d'offre $b^{*}(\cdot)$ soit croissante du signal, le profit devient (en appliquant la fonction réciproque $b^{*-1}$ de la stratégie d'équilibre):

$$
E \Pi_{i}\left(a_{i}, b_{i}\right)=E\left[\left(V_{i}-b_{i}\right) 1_{\left\{\forall j \neq i, A_{j}<b^{*-1}\left(b_{i}\right)\right\}} \mid A_{i}=a_{i}\right]
$$

Soit $Y=\left(Y_{j}\right)_{j=1, \ldots, n}$, le vecteur des statistiques d'ordre associé au vecteur $A=\left(A_{j}\right)_{j=1, \ldots, n}$ des estimations des offreurs. Le vecteur $Y$ correspond aux valeurs classées dans l'ordre décroissant des éléments du vecteur $A$ des signaux et l'on a par conséquent $Y_{1}>Y_{2}>\ldots>Y_{n}$. L'événement "les $n-1$ autres signaux sont inférieurs à $b^{*-1}\left(b_{i}\right)$ " est équivalent à l'événement "le 
deuxième plus grand signal parmi $n$ est inférieur à $b^{*-1}\left(b_{i}\right)$ " et s'exprime par conséquent: $Y_{2}<b^{*-1}\left(b_{i}\right)$.

Définissons aussi l'espérance conditionnelle $\nu\left(y_{1}, y_{2}\right)$ de la valeur de l'objet étant données les deux estimations individuelles les plus fortes $Y_{1}=y_{1}$ et $Y_{2}=y_{2}$. Elle s'exprime:

$$
\nu\left(y_{1}, y_{2}\right)=E\left[V_{1} \mid Y_{1}=y_{1} \& Y_{2}=y_{2}\right]
$$

L'expression du profit espéré devient:

$$
E \Pi_{i}\left(a_{i}, b_{i}\right)=E\left[\left(\nu\left(a_{i}, Y_{2}\right)-b_{i}\right) 1_{\left\{Y_{2}<b^{*-1}\left(b_{i}\right)\right\}} \mid Y_{1}=a_{i}\right]
$$

En notant $F_{Y_{2}}\left(\cdot \mid y_{1}\right)$ la fonction de répartition de $Y_{2}$ sachant la réalisation de $y_{1}$ et $f_{Y_{2}}\left(\cdot \mid y_{1}\right)$ la densité associée, on obtient l'expression équivalente:

$$
E \Pi_{i}\left(a_{i}, b_{i}\right)=\int_{\underline{a}}^{b^{*-1}\left(b_{i}\right)}\left(\nu\left(a_{i}, y\right)-b_{i}\right) d F_{Y_{2}}\left(y \mid a_{i}\right)
$$

où $\underline{a}$ est la plus petite valeur que peuvent prendre les signaux individuels $A_{i}$.

En supposant que $b^{*}(\cdot)$ soit différentiable, une condition nécessaire pour que $b^{*}(\cdot)$ soit une stratégie d'équilibre symétrique est que:

$$
\left.\frac{\partial \Pi_{i}\left(a_{i}, b_{i}\right)}{\partial b_{i}}\right|_{b_{i}=b^{*}\left(a_{i}\right)}=0
$$

qui donne l'équation différentielle linéaire du premier ordre:

$$
b^{* \prime}(y)=\left[\nu(y, y)-b^{*}(y)\right] \frac{f_{Y_{2}}(y \mid y)}{F_{Y_{2}}(y \mid y)}
$$

En posant:

$$
L\left(y \mid a_{i}\right)=\exp \left(-\int_{y}^{a_{i}} \frac{f_{Y_{2}}(a \mid a)}{F_{Y_{2}}(a \mid a)} d a\right)
$$

cette équation est équivalente à :

$$
\frac{d}{d y}\left[b^{*}(y) L\left(y \mid a_{i}\right)\right]=\nu(y, y) \frac{d}{d y}\left[L\left(y \mid a_{i}\right)\right]
$$

qui admet pour solution (en remarquant que $b^{*}(\underline{a})=\nu(\underline{a}, \underline{a})$ et $L\left(a_{i} \mid a_{i}\right)=1$ ):

$$
b^{*}\left(a_{i}\right)=\left[\nu(\underline{a}, \underline{a}) L\left(\underline{a} \mid a_{i}\right)+\int_{\underline{a}}^{a_{i}} \nu(y, y) d L\left(y \mid a_{i}\right)\right]
$$

et peut aussi s'écrire:

$$
b^{*}\left(a_{i}\right)=\nu\left(a_{i}, a_{i}\right)-\int_{\underline{a}}^{a_{i}} L\left(y \mid a_{i}\right) d \nu(y, y)
$$

Le résultat de Milgrom et Weber s'énonce par conséquent: 
Proposition 1 (Milgrom et Weber, 1982) : Sous les hypothèses de symétrie et d'affiliation, en supposant que le prix de réservation du vendeur soit inférieur à a , la stratégie d'équilibre symétrique est donnée par (2) et (3) ou (2) et (4).

Nous allons considérer dans l'application qui suit une famille particulière de distributions multivariées présentant de faibles corrélations due à Gouriéroux et Jouneau. Nous présenterons tout d'abord l'analyse dans le cas de valeurs privées, puis nous considérerons celui de valeur commune.

\subsection{Hypothèse de valeurs privées}

Dans le cas de valeurs privées, nous pouvons négliger la valeur intrinsèque de l'objet $C$ pour nous concentrer sur la distribution des signaux-valeurs privées $\left(A_{i}\right)_{i=1 \ldots n}$. La distribution multivariée $F\left(a_{1}, \ldots, a_{n}\right)$ proposée par Gouriéroux et Jouneau est construite à partir de distributions univariées $F_{i}\left(a_{i}\right)$ suivant la formule:

$$
F\left(a_{1}, \ldots, a_{n}\right)=\left(1+\sum_{i<j} r_{i, j} S_{i}\left(a_{i}\right) S_{j}\left(a_{j}\right)\right) \prod_{j=1}^{n} F_{j}\left(a_{j}\right)
$$

où $S_{i}(a) \equiv 1-F_{i}(a)$ et où les $r_{i, j}, i<j$ sont des nombres réels tels que $\sum_{i<j}\left|r_{i, j}\right| \leq 1$ (cette condition garantit que $0 \leq F \leq 1$ ). Ces paramêtres introduisent une dépendance entre les distributions des signaux $A_{i}$ et $A_{j}$ et sont par conséquent en relation étroite avec les coefficient de corrélation (voir proposition suivante). En particulier, on retrouve le cas d'indépendance en posant $r_{i, j}=0$.

Sous les hypothèse de symétrie et d'affiliation mentionnées plus haut, nous avons $F_{i}(\cdot)=F(\cdot)$ pour tout $i$ et $r_{i, j}=r \geq 0$ pour tout couple $(i, j)$, $i<j$. La condition $\sum_{i<j}\left|r_{i, j}\right| \leq 1$ s'exprime alors $r<2 /(n(n-1))$ et la distribution multivariée devient:

$$
F\left(a_{1}, \ldots, a_{n}\right)=\left(1+r \sum_{i<j} S\left(a_{i}\right) S\left(a_{j}\right)\right) \prod_{j=1}^{n} F\left(a_{j}\right)
$$

La proposition suivante présente un certain nombre de propriétés de ces fonctions. ${ }^{3}$

Proposition 2 (Gouriéroux et Jouneau, 1994) La distribution (5) vérifie les propriétés suivantes

\footnotetext{
${ }^{3}$ Pour d'autres exemples et propriétés, voir Gouriéroux et Jouneau, 1994.
} 
(i) La distribution du couple $\left(A_{i}, A_{j}\right)$ est donnée par

$$
F\left(a_{i}, a_{j}\right)=\left(1+r S\left(a_{i}\right) S\left(a_{j}\right)\right) F\left(a_{i}\right) F\left(a_{j}\right)
$$

(ii) La corrélation du couple $\left(A_{i}, A_{j}\right)$ s'exprime

$$
\rho \equiv \operatorname{Cov}\left[A_{i}, A_{j}\right] / \sigma^{2}=4 r E^{2}[T H(T)]
$$

où $\sigma$ est l'écart type de $A, T$ la variable centrée réduite associée à $A$ et $H(\cdot)$ la distribution de $T$.

(iii) On $a$

$$
\rho<\frac{8}{n(n-1)} E^{2}[T H(T)]
$$

et dans le cas de distributions des $A_{i}$ normales, il vient

$$
\rho<\frac{2}{n(n-1) \pi}
$$

Preuve. voir Gouriéroux et Jouneau, 1994.

La corrélation entre deux valeurs-signaux $\left(A_{i}, A_{j}\right)$ donnée en (ii) est donc une fonction linéaire du facteur de dépendance $r$. Comme souligné en (iii), cette famille de distributions n'autorise que de faibles corrélations entre les variables, ces corrélations étant d'autant plus faibles que le nombre de variables aléatoires est élevé. Le point (i) fait apparaître que la distribution d'un sous vecteur de variables s'exprime de manière très simple à partir de (5). Cela reste le cas pour les lois marginales et il est par ailleurs possible de spécifier les distributions des différentes statistiques d'ordre nécessaires à l'obtention des fonctions d'offre. En particulier, la distribution $F_{1}$ du premier des signaux $Y_{1}$ au niveau $y_{1}$ s'obtient de la manière suivante. Par définition:

$$
\begin{aligned}
F_{1}\left(y_{1}\right) & =P\left[\bigcap_{i}\left(A_{i} \leq y_{1}\right)\right]=\left(1+r \sum_{i<j} S\left(y_{1}\right)^{2}\right) F\left(y_{1}\right)^{n} \\
& =\left(1+\frac{n(n-1)}{2} r S\left(y_{1}\right)^{2}\right) F\left(y_{1}\right)^{n}
\end{aligned}
$$

et par conséquent:

$$
\begin{aligned}
f_{1}\left(y_{1}\right) & =\frac{d F_{1}\left(y_{1}\right)}{d y_{1}}=n F\left(y_{1}\right)^{n-1} f\left(y_{1}\right)\left(1+\frac{n-1}{2} r S\left(y_{1}\right)\left(n S\left(y_{1}\right)-2 F\left(y_{1}\right)\right)\right) \\
& =n F\left(y_{1}\right)^{n-1} f\left(y_{1}\right)\left(1+\frac{n-1}{2} r S\left(y_{1}\right)\left((n+2) S\left(y_{1}\right)-2\right)\right)
\end{aligned}
$$


La distribution $F_{1,2}\left(y_{1}, y_{2}\right)$ du couple des deux meilleurs signaux $\left(Y_{1}, Y_{2}\right)$ aux niveaux $\left(y_{1}, y_{2}\right)$ s'obtient de manière semblable. Par définition:

$$
\begin{aligned}
F_{1,2}\left(y_{1}, y_{2}\right) & =P\left[\bigcup_{i}\left(\bigcap_{j \neq i}\left(A_{j} \leq y_{2}\right) \bigcap\left(y_{2}<A_{i} \leq y_{1}\right)\right)\right] \\
& =P\left[\bigcup_{i}\left(\bigcap_{j \neq i}\left(A_{j} \leq y_{2}\right) \bigcap\left(A_{i} \leq y_{1}\right)\right)\right]-P\left[\bigcup_{i} \bigcap_{j}\left(A_{j} \leq y_{2}\right)\right]
\end{aligned}
$$

avec

$$
\begin{aligned}
& P\left[\bigcup_{i}\left(\bigcap_{j \neq i}\left(A_{j} \leq y_{2}\right) \bigcap\left(A_{i} \leq y_{1}\right)\right)\right] \\
& =n F\left(y_{1}\right) F\left(y_{2}\right)^{n-1}\left(1+r \sum_{j \neq i} S\left(y_{1}\right) S\left(y_{2}\right)+r \sum_{k<j, k \neq i} S\left(y_{2}\right)^{2}\right) \\
& =n\left(1+r(n-1) S\left(y_{1}\right) S\left(y_{2}\right)+r S\left(y_{2}\right)^{2}(n-1)(n-2) / 2\right) F\left(y_{1}\right) F\left(y_{2}\right)^{n-1}
\end{aligned}
$$

et

$$
\begin{aligned}
P\left[\bigcup_{i} \bigcap_{j}\left(A_{j} \leq y_{2}\right)\right] & =n P\left[\bigcap_{j}\left(A_{j} \leq y_{2}\right)\right] \\
& =n F_{1}\left(y_{2}\right)
\end{aligned}
$$

Ces expressions nous permettent d'obtenir les stratégies d'équilibre. Le lecteur intéressé par les différentes étapes de ces calculs peut les trouver en annexe. Dans ce qui suit, nous nous contenterons de placer dans le corps du texte uniquement les quelques formules nécessaires à l'analyse. En particulier, on a le résultat suivant:

Proposition 3 La fonction définie par (2) s'exprime

$$
L\left(y \mid a_{i}\right)=\frac{F(y)^{n-1}}{F\left(a_{i}\right)^{n-1}} \exp \left(\int_{y}^{a_{i}} \frac{r(n-1)(n S(a)-1) f(a)}{1+r(n-1)\left(\frac{n+2}{2} S(a)-1\right) S(a)} d a\right)
$$

Preuve. Voir annexe.

Cette expression est toutefois peu commode et ne permet pas une spécification simple de la fonction d'offre. On peut cependant utiliser le fait que le parametre $r$ est faible pour parvenir à des approximations simples de 
la fonction d'offre. En effet, pour de faibles valeurs de $r$, cette expression devient:

$$
\begin{aligned}
L\left(y \mid a_{i}\right) & \approx \frac{F(y)^{n-1}}{F\left(a_{i}\right)^{n-1}}\left(1+r \int_{y}^{a_{i}}(n-1)(n S(a)-1) f(a) d a\right) \\
& =\frac{F(y)^{n-1}}{F\left(a_{i}\right)^{n-1}}\left(1-r \frac{n-1}{2 n}\left[(n S(a)-1)^{2}\right]_{y}^{a_{i}}\right) \\
& =\frac{F(y)^{n-1}}{F\left(a_{i}\right)^{n-1}}\left(1-r \frac{n-1}{2 n}\left(\left(n S\left(a_{i}\right)-1\right)^{2}-(n S(y)-1)^{2}\right)\right) \\
& =\frac{F(y)^{n-1}}{F\left(a_{i}\right)^{n-1}}\left(1-r(n-1)\left(\frac{S(y)+S\left(a_{i}\right)}{2}-\frac{1}{n}\right)\left(S\left(a_{i}\right)-S(y)\right)\right)
\end{aligned}
$$

La stratégie d'équilibre peut donc être approximée par

$$
\begin{aligned}
b_{r}^{*}\left(a_{i}\right) \approx & a_{i}-\frac{\int_{\underline{a}}^{a_{i}} F(y)^{n-1} d y}{F\left(a_{i}\right)^{n-1}} \\
& -r(n-1) \int_{\underline{a}}^{a_{i}} \frac{F(y)^{n-1}}{F\left(a_{i}\right)^{n-1}}\left(\frac{S(y)+S\left(a_{i}\right)}{2}-\frac{1}{n}\right)\left(S(y)-S\left(a_{i}\right)\right) d y \\
\equiv & b_{0}^{*}\left(a_{i}\right)-r(n-1) C\left(a_{i}\right)
\end{aligned}
$$

La stratégie d'équilibre comprend deux termes. Le premier correspond à la fonction d'offre sans corrélation $b_{0}^{*}(\cdot)=a_{i}-\int_{\underline{a}}^{a_{i}} F(y)^{n-1} d y / F\left(a_{i}\right)^{n-1}$. Le second, paramétré par $r$, rend compte des changements induits par les corrélations. En particulier, comme pour tout $y \leq a_{i}$, on a $S(y) \geq S\left(a_{i}\right)$, le signe du terme sous l'intégrale définissant $C\left(a_{i}\right)$ est donné par celui de $\left(S(y)+S\left(a_{i}\right)\right) / 2-1 / n$. Pour des signaux faibles, tels que $S\left(a_{i}\right) \geq 1 / n$, $C\left(a_{i}\right)$ est sans ambiguïté positif et $b_{r}^{*}\left(a_{i}\right)<b_{0}^{*}\left(a_{i}\right)$. Pour des signaux élevés, et pour certaines distributions, cela peut ne plus être vrai. Ainsi, dans le cas d'une distribution de référence $F$ uniforme (sur un support normalisé $[0,1]$ ), $C\left(a_{i}\right)$ est négatif si $a_{i}>(n+2)(n-1) /(n(n+1))$ (voir annexe). Dans ce cas et pour $n=2$, on a par conséquent $b_{r}^{*}\left(a_{i}\right)>b_{0}^{*}\left(a_{i}\right)$ si $a_{i}>2 / 3$ (c'est-àdire $2 / 3$ de la valeur maximale). Ce seuil croît rapidement avec le nombre d'offreurs: pour $n=4$, seules des valeurs privées au-dessus de $9 / 10$ de la valeur maximale conduisent à des offres plus grandes que lors d'absence de corrélation. ${ }^{4}$ L'allure générale des fonctions d'offre est représentée figure 1.

L'offre d'un individu ayant une évaluation faible est en-dessous de l'offre sans affiliation $b_{\mathbf{0}}^{*}(\cdot)$ : comme il existe des corrélations positives entre les

\footnotetext{
${ }^{4}$ Observons que ce seuil évolue plus rapidement que l'espérance de la statistique d'ordre du premier signal parmi $n$ correspondant au cas d'indépendance. Cette dernière est en effet donné par $n /(n+1)$ et n'est égal à ce seuil que pour $n=2$.
} 


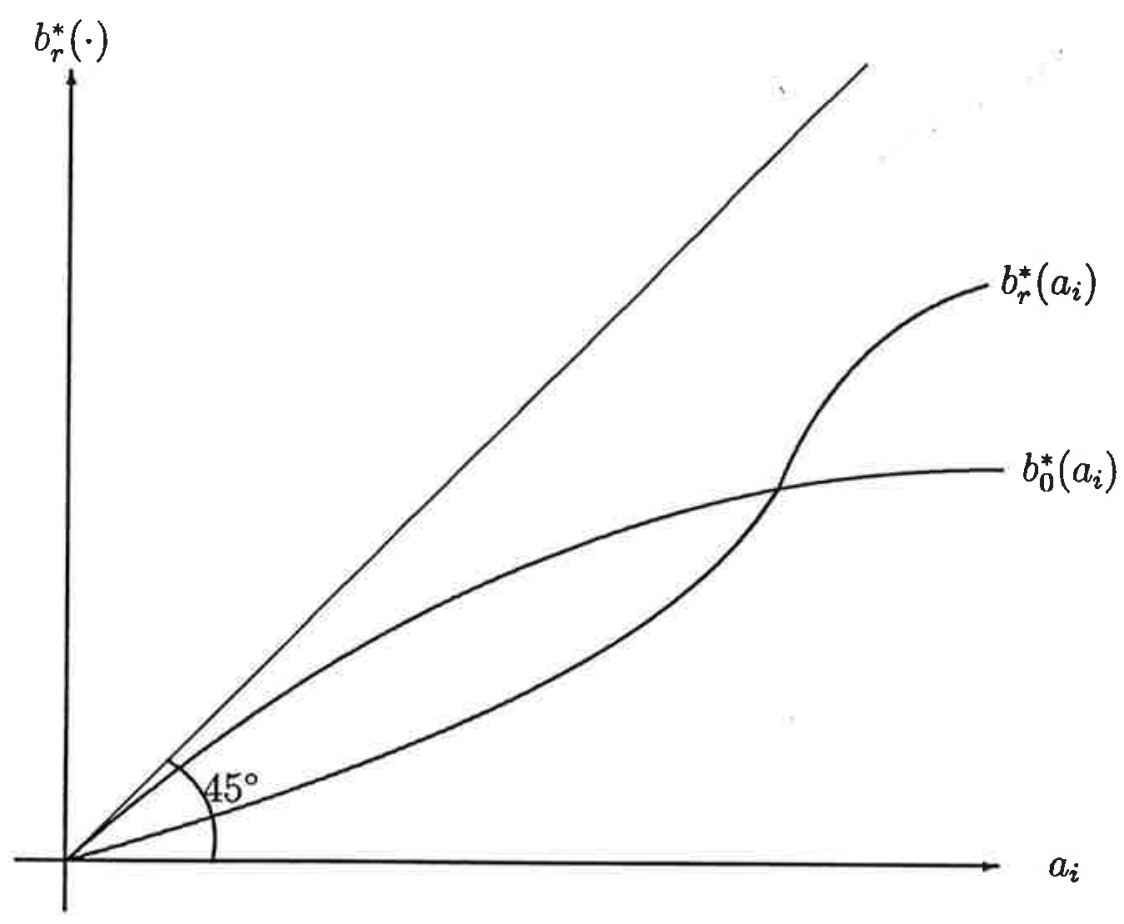

Figure 1: fonctions d'offre - Valeurs privées

valeurs, il est en effet fort probable que les autres signaux soient faibles, de sorte que l'individu peut baisser son offre par rapport au cas d'indépendance. Le phénomène inverse peut se produire dans le cas d'une évaluation élevée, c'est-à-dire pour une valeur privée largement supérieure à l'espérance de la distribution de référence: si la valeur privée est proche de cette espérance et qu'elle est gagnante, cela signifie que toutes les autres valeurs sont probablement inférieures à l'espérance, et donc finalement que les évaluations sont dans leur ensemble relativement faibles. Une évaluation gagnante forte est par conséquent une évaluation largement au-dessus de l'espérance, et cet écart est d'autant plus grand que le nombre d'offreurs est important.

\subsection{Hypothèse de valeur commune}

Nous allons maintenant considérer que les individus reçoivent un signal de la vraie valeur $c$. Il est facile d'étendre à ce cadre la distribution multivariée (5), en considérant qu'il s'agit d'une distribution conditionnelle qui s'écrit:

$$
F\left(a_{1}, \ldots, a_{n} \mid c\right)=\left(1+r \sum_{i<j} S\left(a_{i} \mid c\right) S\left(a_{j} \mid c\right)\right) \prod_{j=1}^{n} F\left(a_{j} \mid c\right)
$$


Dans l'application qui suit nous ferons, à la suite de Thiel (1988), l'hypothèse d'un a priori diffus: la distribution de $C$ est supposée constante. ${ }^{5}$ Nous supposerons aussi que la distribution de référence $F\left(a_{i} \mid c\right)$ est une loi normale, d'espérance égale à la valeur commune du bien $c$, dont la densité s'exprime :

$$
f\left(a_{i} \mid c\right)=\frac{1}{\sigma \sqrt{2 \pi}} \exp \left(-\frac{1}{2}\left(\frac{a_{i}-c}{\sigma}\right)^{2}\right)=\frac{1}{\sigma} \phi(t)
$$

où $t=\left(a_{i}-c\right) / \sigma$ est la variable centrée réduite et $\phi(\cdot)$ la densité d'une loi normale centrée réduite (dont on notera $\Phi$ la distribution). Sous ces hypothèses, l'offre d'un individu est une fonction linéaire de son signal dans le cas d'indépendance. La proposition suivante montre que cette propriété est conservée dans notre cadre. (Comme précédemment, les expressions apparaissant dans le texte sont issues de calculs donnés en annexe.)

Proposition 4 La stratégie d'équilibre s'exprime:

$$
b_{r}^{*}\left(a_{i}\right)=a_{i}-\sigma \alpha_{n}(r)
$$

où

avec

$$
\alpha_{n}(r)=\frac{E_{n}\left[t^{2}\right]+r n(n-1) \psi_{n}\left(t^{2}-1\right) / 2}{E_{n}[t]+r n(n-1) \psi_{n}(t) / 2}
$$

$$
\psi_{n}(t)=E_{n}[t]-\frac{2(n-1)(n+2)}{n(n+1)} E_{n+1}[t]+\frac{(n-1)(n+2)+2 n}{(n+1)(n+2)} E_{n+2}[t]
$$

et $E_{l}[t]=\int_{-\infty}^{+\infty} t d \Phi(t)^{l}$.

Preuve. Voir annexe.

Observons que la stratégie d'équilibre donnée par (9) reste linéaire et que les corrélations n'affectent que le terme constant $\alpha_{n}(r)$, qui est une fonction non-linéaire de $r$. Ce dernier terme peut cependant être approximé par une fonction linéaire de $r$, ce paramètre ne prenant que de faibles valeurs. Un développement au premier ordre donne:

$$
\begin{aligned}
b_{r}^{*}\left(a_{i}\right) & \approx a_{i}-\sigma\left(\alpha_{n}(0)+r \alpha_{n}^{\prime}(0)\right) \\
& =a_{i}-\sigma \frac{E_{n}\left[t^{2}\right]}{E_{n}[t]}-\sigma r \frac{n(n-1)}{2} \frac{\psi_{n}(t)}{E_{n}[t]}\left(\frac{\psi_{n}\left(t^{2}-1\right)}{\psi_{n}(t)}-\frac{E_{n}\left[t^{2}\right]}{E_{n}[t]}\right) \\
& \equiv b_{0}^{*}\left(a_{i}\right)-r \sigma \kappa_{n}
\end{aligned}
$$

${ }^{5}$ Pour un commentaire critique de l'approche de Thiel, voir Levin et Smith, 1991. 
où $b_{0}^{*}(\cdot)$ est la fonction d'offre sans corrélation. Observons que pour $r$ et $\sigma$ donnés, cette approximation ne diffère de la stratégie $b_{0}^{*}(\cdot)$ que par une constante, du signe contraire de $\kappa_{n}$. Le tableau suivant présente quelques valeurs de $\kappa_{n}$ en fonction du nombre d'offreurs pour $r$ et $\sigma$ donnés.

\begin{tabular}{ccccccccccc}
\hline \hline$n$ & 5 & 6 & 7 & 8 & 9 & 10 & 15 & 20 & 25 & 30 \\
\hline$\kappa_{n}$ & -0.29 & -0.26 & -0.24 & -0.21 & -0.19 & -0.18 & -0.12 & -0.09 & -0.07 & -0.06 \\
\hline \hline
\end{tabular}

Tableau 1: Coefficient affectant la stratégie d'équilibre en fonction du nombre d'offeurs (approximation)

Il apparaît à la lecture du tableau 1 que le terme $-r \sigma \kappa_{n}$ est positif mais que sa valeur absolue est d'autant plus faible que le nombre d'offreurs est grand. La corrélation des signaux induit par conséquent un comportement d'offre plus agressif que la situation sans corrélation. L'allure générale des fonctions d'offre est représentée figure 2.

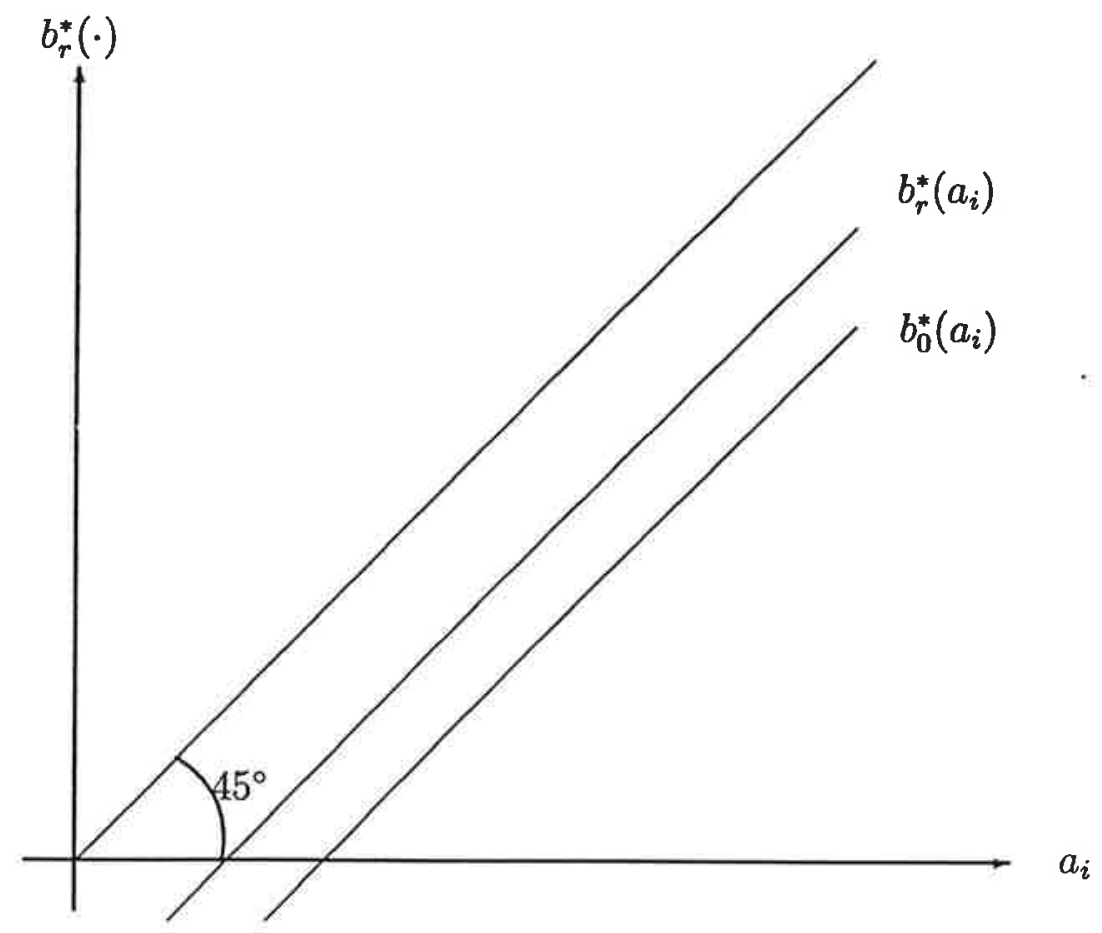

Figure 2: fonctions d'offre - Valeur commune

Comme souligné plus haut, les fonctions d'offre sous les hypothèses de Thiel sont des fonctions linéaires du signal. Dans la figure 2, nous avons représenté essentiellement la partie positive de ces offres: la spécification 
retenue permet en effet des évaluations et donc des offres négatives, la fonction de distribution de référence prenant ses valeurs sur tout $\mathbb{R}{ }^{6}$ La fonction d'offre avec corrélations $b_{r}^{*}$ se situe entre la première bissectrice et la fonction d'offre sans corrélation $b_{0}^{*}$. Par conséquent pour un même signal $a_{i}$, l'offre avec corrélation sera supérieure à l'offre sans corrélation, et cela quelque soit le niveau de ce signal, à la différence du cas valeurs privées.

\section{Méthodes d'estimation}

Nous allons à présent étendre la méthode d'estimation présentée dans Bourgeon et Le Roux (1995) au cas de la valeur commune. L'extension au cas "valeurs privées" est rendue difficile par le fait que le théorème d'équivalence revenu ne s'applique pas dans le cas d'affiliation (voir Milgrom et Weber, 1982). Par contre, le fait que l'offre d'équilibre reste linéaire dans le cas "valeur commune" permet d'adopter la même démarche que celle suivie dans notre précédent article, qui repose essentiellement sur une simple régression linéaire. La généralisation de cette démarche est en particulier permise par la méthode des moindres carrés asymptotiques (Gouriéroux, Monfort et Trognon, 1985). Cette méthode s'effectue en deux étapes, la première consistant en une régression linéaire qui permet d'estimer une partie des paramètres d'intérêt et un certain nombre de variables auxiliaires, la seconde étape consistant en la résolution d'un système d'équations faisant intervenir les variables auxiliaires estimées en première étape afin d'évaluer les paramètres d'intérêt non encore spécifiés.

Dans un deuxième temps, afin de confirmer les résultats obtenus et du fait de la forte sensibilité de l'estimation du coefficient $r$ aux niveaux des paramètres auxiliaires de première étape, nous procédons à une estimation directe de l'ensemble des paramètres d'intérêt par une méthode de moindres carrés non-linéaires, en utilisant comme variables endogènes les offres gagnantes et les deuxièmes plus grandes offres, ainsi que les carrés de celles-ci, que l'on peut régresser sur les moments théoriques non centrés du deuxième ordre. ${ }^{7}$

\footnotetext{
${ }^{6}$ On pourrait se restreindre à des distributions prenant leurs valeurs exclusivement sur $\mathbb{R}_{+}$et conserver cette propriété de linéarité (voir, par exemple, Wilson 1992), au prix d'un plus grand effort de calcul. L'offre est alors proportionnelle au signal reçu. On montre cependant dans Bourgeon et Le Roux (1995) que les estimations obtenues avec la loi normale et la loi log-normale sont identiques, de sorte que l'on peut se limiter au cas le plus simple à traiter.

${ }^{7}$ D'autre démarches sont naturellement possibles. Voir en particulier la méthode des moments généralisés (Hansen, 1982).
} 


\subsection{Estimation en deux étapes : moindres carrés asymp- totiques}

D'après (9), l'offre gagnante s'écrit:

$$
b^{w}=a^{w}-\sigma \alpha_{n}(r)
$$

où $a^{w}$ est la réalisation du signal gagnant $Y_{1}$ et l'on a aussi :

$$
b_{(2)}=y_{2}-\sigma \alpha_{n}(r)
$$

Ces expressions nous permettent en particulier d'obtenir les résultats suivants:

Proposition 5 L'espérance et la variance de l'offre gagnante et de la seconde plus grande offre s'expriment:

$$
\begin{gathered}
E\left[B^{w} \mid C\right]=c-\sigma \alpha_{n}(r)+\sigma \varphi_{1}(t, r) \\
E\left[B_{(2)} \mid C\right]=c-\sigma \alpha_{n}(r)+\sigma \varphi_{2}(t, r) \\
V\left[B^{w} \mid C\right]=\sigma^{2}\left(\varphi_{1}\left(t^{2}, r\right)-\varphi_{1}(t, r)^{2}\right) \\
V\left[B_{(2)} \mid C\right]=\sigma^{2}\left(\varphi_{2}\left(t^{2}, r\right)-\varphi_{2}(t, r)^{2}\right)
\end{gathered}
$$

où:

$$
\varphi_{1}(t, r)=E_{n}[t]+r \frac{n(n-1)}{2}\left(E_{n+2}[t]-2 E_{n+1}[t]+E_{n}[t]\right)
$$

et

$$
\begin{aligned}
\varphi_{2}(t, r)= & E_{(2 \mid n)}[t]+r \frac{n(n-1)}{2}\left((n-2) E_{n-1}[t]-3(n-3) E_{n}[t]\right. \\
& \left.+3(n-4) E_{n+1}[t]-(n-5) E_{n+2}[t]\right)
\end{aligned}
$$

avec :

$$
E_{(2 \mid n)}[t]=\int_{-\infty}^{+\infty} t n(n-1) \Phi(t)^{n-2}(1-\Phi(t)) \phi(t) d t
$$

Preuve. Voir annexe.

Dans le cadre de l'hypothèse de valeur commune, et selon l'équation (10), l'expression de l'espérance de l'offre gagnante s'exprime, pour chaque enchère $l$ :

$$
E\left[B_{l}^{w} \mid C_{l}\right]=c_{l}-\sigma \alpha_{n}(r)+\sigma \varphi_{1}(t, r)
$$


Supposons que l'espérance $c_{l}$ des signaux $a_{l}$ soit de la forme:

$$
c_{l}=\alpha_{0}+z_{l}^{\prime} \delta
$$

où $\alpha_{0}$ est une constante, $z_{l}=\left(1, z_{l}^{\prime}\right)$ un vecteur de variables explicatives, et $\delta$ un vecteur de paramètres. L'offre gagnante devient:

$$
b_{l}^{w}=z_{l}^{\prime} \delta+\alpha_{1}+\varepsilon_{1 l}
$$

où les $\varepsilon_{1 l}$ sont supposés normaux, d'espérance nulle, indépendamment et identiquement distribués (i.i.d.) et où :

$$
\alpha_{1}-\alpha_{0}+\sigma\left[\varphi_{1}(t, r)-\alpha_{n}(r)\right]=0
$$

Pour un nombre d'offreurs $n$ donné, et pour un coefficient $r$ constant, l'expression de l'offre gagnante est une fonction linéaire en $z_{l}^{\prime}$. De même, d'après l'équation (11), la deuxième plus grande offre est aussi une fonction linéaire en $z_{l}^{\prime}$, pour $n$ et $r$ donnés, qui s'exprime, pour chaque enchère $l$ :

$$
b_{(2) l}=z_{l}^{\prime} \delta+\alpha_{2}+\varepsilon_{2 l}
$$

où les $\varepsilon_{2 l}$ sont supposés normaux, d'espérance nulle et i.i.d. et où :

$$
\alpha_{2}-\alpha_{0}+\sigma\left[\varphi_{2}(t, r)-\alpha_{n}(r)\right]=0
$$

La première étape de la méthode des moindres carrés asymptotiques consiste à effectuer une estimation simultanée des équations (13) et (15), par une méthode de moindres carrés avec contraintes sur les paramètres (contrainte à l'égalité des coefficients du vecteur $\delta$ apparaissant dans les deux équations). Cette estimation permet de caractériser les effets des variables explicatives $z_{l}^{\prime}$ sur la valeur commune $c_{l}$ (estimation du vecteur de paramètres $\delta$ ) et de calculer les constantes $\alpha_{1}$ et $\alpha_{2}$. A partir des séries de résidus obtenues, elle permet aussi d'obtenir une estimation des variances résiduelles $\hat{\sigma}_{B^{w}}^{2}$ et $\hat{\sigma}_{B_{(2)}}^{2}$, qui, sous les hypothèses de normalité et d'indépendance des termes d'erreurs, sont distribués selon une loi du $\chi^{2}$. Elle n'autorise cependant pas l'identification directe de la constante $\alpha_{0}$, de l'écart-type $\sigma$ de la loi définissant la distribution latente des signaux, ni du coefficient $r$.

La seconde étape consiste donc à utiliser les estimateurs des paramètres $\hat{\alpha}_{1}, \hat{\alpha}_{2}$ et $\hat{\sigma}_{B^{w}}$ (paramètres auxiliaires) pour déterminer les coefficients manquants de la distribution latente $\alpha_{0}, \sigma$ et $r$ (paramètres d'intérêt). En particulier, à partir de la variance de l'offre gagnante donnée par (12), et de l'estimation $\hat{\sigma}_{B^{w}}^{2}$ de la variance résiduelle des offres gagnantes, l'écart-type estimé $\hat{\sigma}$ doit vérifier la contrainte: 


$$
\hat{\sigma}_{B^{w}}^{2}-\hat{\sigma}^{2}\left(\varphi_{1}\left(t^{2}, r\right)-\varphi_{1}(t, r)^{2}\right)=0
$$

La résolution du système d'équations (14), (16) et (17), appelé système d'équations estimantes, permet donc d'obtenir indirectement les estimations $\hat{\alpha}_{0}, \hat{\sigma}$ et $\hat{r}$ des paramètres de la distribution latente à partir des estimations $\hat{\alpha}_{1}, \hat{\alpha}_{2}$ et $\hat{\sigma}_{B^{w}}$ des paramètres issus de l'étape de régression. La méthode des moindre carrés asymptotiques permet d'effectuer une telle estimation par résolution du problème:

$$
\min _{a}{ }^{t} G(\hat{b}, a) \hat{S}_{0} G(\hat{b}, a)
$$

où $\hat{b}=\left(\hat{\alpha}_{1}, \hat{\alpha}_{2}, \hat{\sigma}_{B^{w}}\right)$ est le vecteur des paramètres auxiliaires, $a=\left(\alpha_{0}, \sigma, r\right)$ le vecteur des paramètres d'intêret, $G(\hat{b}, a)$ le système des contraintes estimantes défini par les membres de gauche des équations (14), (16) et (17), ${ }^{t} G(\hat{b}, a)$ la transposée du vecteur $G(\hat{b}, a)$, et où $S_{0}$ est la matrice définie par:

$$
S_{0}={ }^{t} \nabla_{b} G \Omega \nabla_{b} G
$$

avec: $\nabla_{b} G$ la matrice jacobienne du vecteur $G$ par rapport aux paramètres $b$, et:

$$
\Omega=V[\hat{b}]=\left(\begin{array}{cc}
V\left[\hat{\alpha}_{1}, \hat{\alpha}_{2}\right] & 0 \\
0 & V\left[\hat{\sigma}_{B^{w}}^{2}\right]
\end{array}\right)
$$

la matrice de variance-covariance des paramètres auxiliaires. La variance $V[\hat{a}]$ des paramètres d'intêret estimée par cette méthode se déduit de celle des paramètres auxiliaires selon la formule (voir Gouriéroux, Monfort et Trognon, 1985):

$$
V[\hat{a}]=\left[{ }^{t} \nabla_{a} G\left[{ }^{t} \nabla_{b} G \Omega \nabla_{b} G\right]^{-1} \nabla_{a} G\right]^{-1}
$$

où $\nabla_{a} G$ est la matrice jacobienne du vecteur $G$ par rapport aux paramètres $a$.

\subsection{Estimation directe}

Cette méthode permet d'estimer en une seule étape l'ensemble des coefficients caractérisant la distribution latente et les corrélations entre offres. Elle consiste simplement à régresser non seulement les séries des variables endogènes en niveau sur leurs espérances mais aussi les séries des carrés sur leurs distributions théoriques, qu'il est facile de spécifier à partir des résultats 
précédents (voir annexe). Le système à estimer s'écrit:

$$
\begin{cases}b_{l}^{w} & =z_{l}^{\prime} \delta+\alpha_{0}+\sigma\left[\varphi_{1}(t, r)-\alpha_{n}(r)\right]+\varepsilon_{1 l} \\ b_{(2) l} & =z_{l}^{\prime} \delta+\alpha_{0}+\sigma\left[\varphi_{2}(t, r)-\alpha_{n}(r)\right]+\varepsilon_{2 l} \\ \left(b_{l}^{w}\right)^{2} & =\left[z_{l}^{\prime} \delta+\alpha_{0}+\sigma\left(\varphi_{1}(r, t)-\alpha_{n}(r)\right)\right]^{2}+\sigma^{2}\left[\varphi_{1}\left(t^{2}, r\right)-\varphi_{1}(t, r)\right]+\varepsilon_{3 l} \\ \left.b_{(2) l}^{2}\right] & =\left[z_{l}^{\prime} \delta+\alpha_{0}+\sigma\left(\varphi_{2}(r, t)-\alpha_{n}(r)\right)\right]^{2}+\sigma^{2}\left[\varphi_{2}\left(t^{2}, r\right)-\varphi_{2}(t, r)\right]+\varepsilon_{4 l}\end{cases}
$$

où les $\varepsilon_{i l}$ sont supposés i.i.d. pour chaque $i=1, \ldots, 4$.

Observons que ce système n'est pas linéaire, faisant intervenir non seulement les carrés des paramètres, mais aussi la fonction $\alpha_{n}(r)$. Cette dernière peut toutefois être linéarisée comme nous l'avons indiqué plus haut, en utilisant le fait que le paramètre $r$ ne peut prendre que de faibles valeurs. De la même manière, après développement des carrés apparaissant dans les moments théoriques du second ordre, on peut, dans une première approche, faire disparaître les termes impliquant un carré du paramètre $r$.

\section{Application empirique}

Le modèle d'enchères avec faibles corrélations des signaux présenté ci-dessus est appliqué aux adjudications de stocks d'intervention de blé tendre effectuées par le Comité de Gestion des Céréales de la Commission Européenne. L'échantillon retenu est constitué de 41 lots homogènes, adjugés en 1991 et 1992. Pour chacun de ces lots, sont disponibles les valeurs de l'offre gagnante et de la deuxième plus grande offre.

\subsection{Estimation en deux étapes}

Comme nous l'avons exposé dans la section précédente, l'estimation simultanée du système:

$$
\begin{aligned}
b_{l}^{w} & =z_{l}^{\prime} \delta+\alpha_{1}+\varepsilon_{1 l} \\
b_{(2) l} & =z_{l}^{\prime} \delta+\alpha_{2}+\varepsilon_{2 l}
\end{aligned}
$$

permet de déterminer les caractéristiques des espérances des signaux (paramètres $\delta$ du tableau 2) et à partir des séries des résidus, les variances résiduelles $\hat{\sigma}_{B^{w}}^{2}$ et $\hat{\sigma}_{B_{(2)}}^{2}$

L'espérance des signaux sur la valeur commune est expliquée de façon satisfaisante par les variables économiques retenues. Hormis les coefficients $\alpha_{1}$ et $\alpha_{2}$ qui dépendent entre autres de la constante de cette espérance, les régressions de la première et de la deuxième offre permettent de caractériser 
l'influence de ces variables sur l'espérance de ces signaux. Les offres ( $b^{w}$ et $\left.b_{(2)}\right)$ sont exprimées en Ecus par tonne. ${ }^{8}$ Le taux de change entre l'Ecu et le dollar américain, monnaie de transaction des échanges de céréales, a donc naturellement un effet positif sur les anticipations individuelles de la valeur $\mathrm{du}$ bien. L'élasticité de l'offre gagnante au taux de change est, au point moyen de l'échantillon, de 1.27: outre l'ajustement mécanique à la variation observée du taux de change, on note un comportement spéculatif lié aux anticipations des variations de ce taux de change.

Le volume de blé tendre exporté par les Etats-Unis simultanément à l'adjudication européenne (en fait, sont prises en compte ici les quantités exportées mensuellement par les Etats-Unis) a un effet négatif sur les offres des opérateurs et donc sur leurs anticipations individuelles. Une augmentation de ces exportations américaines, par exemple, peut indiquer une "agressivité" accrue des Etats-Unis sur le marché mondial (en termes quantitatifs), celle-ci pouvant induire une réaction en termes de prix côté européen (par une baisse des offres, donc du prix de revente qui sera pratiqué sur le marché mondial). L'élasticité des offres européennes à ce volume exporté par les Etats-Unis reste toutefois limitée, puisqu'elle n'est égale qu'à -0.08 au point moyen.

Plus significative dans son ampleur est l'influence du prix réel à l'exportation pratiqué par les Etats-Unis. Le prix utilisé dans l'estimation est le prix à l'exportation fob Golfe du Mexique (qualité HRW), corrigé d'un bonus moyen (subvention américaine à l'exportation de produits agricoles). Ce prix réel à l'exportation pratiqué par les Etats-Unis a un effet que l'on peut qualifier de directeur sur le marché mondial, donc sur les prix européens à l'exportation et par voie de conséquence sur le niveau des subventions desquelles ceux-ci bénéficient. L'élasticité des offres européennes lors des adjudications de stocks, relativement à ce prix européen, est en moyenne de 0.9.

Enfin, compte-tenu de la double vocation des stocks constitués par l'UE (soit être écoulés sur le marché intérieur européen, soit être revendus à l'exportation), le prix pratiqué sur le marché européen est un indicateur de la profitabilité des exportations, relativement aux ventes domestiques. Ce prix intérieur influence donc négativement les offres d'achat de stocks en vue de l'exportation, avec une élasticité de -1.5 au point moyen.

La caractérisation de l'espérance des anticipations individuelles produit un premier ensemble de résultats qui est globalement comparable, quant aux variables influençant significativement les offres des opérateurs et quant aux

\footnotetext{
${ }^{8} \mathrm{~L}$ 'offre formulée par un opérateur correspond au prix de revente sur le marché mondial qu'il souhaite obtenir, déduction faite des marges et des coûts de transport. De plus, l'offre doit inclure une "restitution technique" qui sera reversée à l'opérateur une fois l'exportation réalisée, et qui n'existe que pour des raisons juridiques (cette restitution constitue la base d'application des pénalités, en cas de non respect du contrat, par exemple).
} 


\begin{tabular}{ccc}
\hline \hline Equations & 1ère Offre & 2ème offre \\
\hline Constantes & $\hat{\alpha}_{1}$ & $\hat{\alpha}_{2}$ \\
& 29.71 & 28.65 \\
& $(3.28)$ & $(3.16)$ \\
\hline Variances & $\hat{\sigma}_{B^{w}}^{2}$ & $\hat{\sigma}_{B_{(2)}}^{2}$ \\
& 0.455 & 2.807 \\
[écarts-types estimés] & {$[0.08]$} & {$[0.47]$} \\
\hline Variables $\left(z_{l}\right)$ & Paramètres $(\delta)$ \\
\hline Ecu/Usd & 13.777 \\
Qeu & $(7.11)$ \\
(taux de change Ecu/dollar US) & -6.525 \\
(exportations des Etats-Unis) & $(-11.79)$ \\
Peu & \multicolumn{2}{c}{0.657} \\
(prix réel à l'exportation des Etats-Unis) & $(23.88)$ \\
Pue & -0.080 \\
(prix intérieur européen) & $(-16.07)$ \\
\hline Ecart quadratique moyen & $0.99 \%$ \\
\hline \hline (statistiques de Student entre parenthèses) & $2.49 \%$ \\
\hline
\end{tabular}

Tableau 2: Estimations des caractéristiques de l'espérance des anticipations individuelles

signes de ces effets, à ceux obtenus dans Bourgeon et Le Roux (1995), avec une spécification ne prenant pas en compte l'hypothèse de corrélation entre offres, et sur un échantillon similaire. Sur le plan empirique, les améliorations s'enregistrent dans la seconde phase d'estimation, dont les résultats sont présentés dans ce qui suit.

Les estimations des constantes $\alpha_{1}$ et $\alpha_{2}$ et de la variance $\sigma_{B^{w}}^{2}$ obtenues ci-dessus permettent de résoudre le système des équations estimantes (14), (16) et (17) suivant la méthode des moindres carrés asymptotiques, cela pour un nombre d'offreurs $n$ donné. Compte-tenu des informations sur le nombre d'opérateurs soumissionnant lors des adjudications de stocks d'intervention (cf., par exemple, Debatisse, 1984), nous supposons que ce nombre $n$ est le même pour chaque adjudication, égal à 15 (les résultats ne sont pas sensiblement modifiés pour des valeurs différentes de 15). Pour $n$ fixé à 15 , la résolution de ce système permet de déterminer les autres caractéristiques de la distribution latente des signaux sur la valeur commune (tableau 3).

Les résultats relatifs à la caractérisation de la distribution des signaux (i.e. 


\begin{tabular}{ll}
\hline \hline$\hat{\sigma}^{2}$ & 1.075 \\
(écart-type des signaux) & $(8.88)$ \\
\hline$\hat{r}$ & 0.6428 \\
(facteur de corrélation) & $(3.50)$ \\
\hline$\hat{\alpha}_{0}$ & 27.906 \\
\hline \multicolumn{2}{l}{$(3.08)$} \\
\hline \hline (statistiques de Student entre parenthèses) \\
\hline \hline
\end{tabular}

Tableau 3: Espérance, écart-type des signaux et indicateur de corrélation entre offres estimées

\begin{tabular}{ll}
\hline \hline$\hat{E}\left(b^{w}\right)$ & 63.58 Ecus \\
\hline$\hat{E}\left(b_{(2)}\right)$ & 62.52 Ecus \\
\hline$\hat{c}$ & 63.75 Ecus \\
\hline$\hat{\sigma} / \hat{c}$ & $1.6 \%$ \\
\hline \hline
\end{tabular}

Tableau 4: Valeurs de marché estimées

leur espérance $\alpha_{0}+z_{l}^{\prime} \delta$ et leur écart-type $\sigma$ ) révèle que la valeur commune serait toujours très proche de l'offre gagnante, pour chacun des lots adjugés. La valeur commune estimée s'établit à 63.75 Ecus/tonne en moyenne sur l'échantillon (tableau 4), pour une offre gagnante de 63.58 Ecus/tonne en moyenne également. De plus, la dispersion des signaux perçus par les opérateurs est très faible autour de cette valeur commune $(\hat{\sigma}=1.04 \mathrm{Ecu}$, soit 1.6 $\%$ de la moyenne estimée de la valeur commune).

Par ailleurs, les résultats relatifs à la corrélation des offres exhibent une valeur de $r$ particulièrement élevée (0.64). De plus, alors que les coefficients caractéristiques de la distribution des signaux $\left(\alpha_{0}\right.$ et $\sigma$ pour les résultats de la deuxième étape) sont particulièrement stables (en faisant varier $n$ par exemple), une analyse de sensibilité montre la grande variabilité du coefficient $r$, en fonction des résultats de la première étape. En particulier, cet indicateur de la corrélation entre les offres des opérateurs dépend fortement de l'écart entre les constantes présentes dans les expressions linéaires des espérances de l'offre gagnante et de la deuxième plus grande offre $\left(\alpha_{1}-\alpha_{2}\right)$.

Comme l'indique le tableau 5 , de faibles corrélations sont obtenues pour un écart $\left(\alpha_{1}-\alpha_{2}\right)$ proche de 0.25 , mais des valeurs beaucoup plus élevées en valeur absolue sont observées au voisinage de cet écart. Cette forte instabilité du coefficient $r$ montre qu'il n'est pas possible, à partir des résultats de cette estimation en deux étapes, de conclure sur l'existence d'une corrélation significativement non nulle entre les offres. 


\begin{tabular}{lccccccccc}
\hline \hline$\alpha_{1}-\alpha_{2}$ & 1.034 & 1. & 0.5 & 0.3 & 0.26 & 0.25 & 0.24 & 0.23 & 0.2 \\
\hline$\hat{r}$ & 0.6428 & 0.589 & 0.163 & 0.031 & 0.007 & 0.001 & -0.005 & -0.011 & -0.028 \\
\hline \hline
\end{tabular}

Tableau 5: Sensibilité du coefficient d'affiliation aux niveaux estimés

\subsection{Méthode directe}

La réponse à cette interrogation est fournie par la méthode directe d'estimation que nous avons présentée plus haut, et qui consiste à prendre en compte les moments du deuxième ordre de l'offre gagnante et de la deuxième plus grande offre lors de la phase d'estimation. Pour $n$ donné $(n=15)$, l'estimation simultanée du système (18) conduit à l'identification du vecteur de paramètres $\delta$ et des coefficients $\alpha_{0}, \sigma$, et $r$.

Les résultats d'estimation permettent de conclure de façon identique quant aux effets des variables explicatives sur les signaux perçus par les offreurs. Les coefficients du vecteur $\delta$ sont en effet proches de ceux obtenus précédemment. De même, la dispersion des signaux autour de la valeur commune est caractérisée par un écart-type estimé proche de celui calculé en deuxième étape de la méthode précédente $(\hat{\sigma} \simeq 1)$. La série ajustée de la valeur commune présente donc ici également une évolution très proche de celle des offres gagnantes, et les anticipations individuelles des opérateurs sont très concentrées autour de cette valeur commune.

L'estimation du coefficient $r$ diffère quant à elle du résultat obtenu par la première méthode $(r=0.5)$. Notons toutefois que cet écart est peu significatif, compte-tenu de la forte instabilité de ce coefficient, observée lors de l'application de cette première méthode. De plus, outre le fait que cette deuxième méthode confirme les enseignements de la première quant à la caractérisation de la valeur commune, l'estimation directe du coefficient $r$ (même si elle est encore particulièrement élevée) permet de conclure que la corrélation entre les offres des opérateurs est significativement positive (l'écart-type estimé du coefficient $r$ étant de 0.2 environ). La spécification retenue n'est toutefois valide que pour de faibles corrélations (cf. supra), et l'estimation réalisée ici conduit à suspecter une corrélation forte entre les comportements d'offre des participants aux adjudications. 


\begin{tabular}{lc}
\hline \hline Constantes & \\
\hline$\hat{\alpha}_{0}$ & 14.047 \\
& $(1.20)$ \\
$\hat{\sigma}$ & 1.005 \\
& $(172)$. \\
$\hat{r}$ & 0.499 \\
& $(2.40)$ \\
\hline Variables $\left(z_{l}\right)$ & Paramètres $(\delta)$ \\
\hline Ecu/Usd & 16.680 \\
(taux de change Ecu/dollar US) & $(6.71)$ \\
Qeu & -6.081 \\
(exportations des Etats-Unis) & $(-12.04)$ \\
Peu & 0.689 \\
(prix réel à l'exportation des Etats-Unis) & $(20.53)$ \\
Pue & -0.085 \\
(prix intérieur européen) & $(-14.66)$ \\
\hline Ecarts quadratiques moyens & \\
\hline$B_{l}^{w}$ & $1.06 \%$ \\
$B_{(2) l}$ & $2.47 \%$ \\
\hline \hline (statistiques de Student entre parentheses) \\
\hline
\end{tabular}

Tableau 6: Estimation directe des coefficients

\section{Conclusion}

La prise en compte de corrélations entre les signaux-valeurs privées dans les modèles d'enchères que nous avons présentés, conduit à des évolutions différentes des comportements d'offre suivant le paradigme retenu: dans le cas de valeurs privées, l'offre est plus faible que dans le cas sans corrélation sur la majeure partie du support des valeurs possibles, et cela est d'autant plus vrai que le nombre d'offreurs est important (même si, il est vrai, la distance entre les deux fonctions tend à diminuer), alors que dans le cas de la valeur commune, les offres avec corrélations se situent sans ambiguitté au-dessus de la fonction d'offre sans affiliation. L'existence de corrélations entre les signauxvaleurs privées vient donc nuancer le phénomène mis en évidence dans notre précédent article: en effet, sous l'hypothèse d'indépendance, l'espérance de l'offre gagnante est, dans le cas du paradigme des valeurs privées, plus élevée que l'espérance de la distribution latente (la moyenne des valeurs privées), 
alors que dans le cas du paradigme de la valeur commune, elle est plus faible que l'espérance de la distribution latente (la moyenne des signaux individuels, qui est aussi la valeur commune). Cette observation nous avait notamment permis de conclure que le comportement des compagnies de négoce sur le marché mondial des céréales relevait plus probablement du paradigme de la valeur commune que de celui des valeurs privées. Avec affiliation des distributions latentes des signaux-valeurs privées, l'écart à l'espérance de la distribution latente des espérances des deux fonctions d'offre se trouve réduit, ce qui conduit à un rapprochement des comportements théoriques (tout du moins en moyenne).

La caractérisation de ces évolutions a été rendue possible par le biais d'une forme très spécifique de distribution multivariée, n'autorisant que de faibles corrélations, mais qui permet d'obtenir des approximations explicites des fonctions d'offre. A partir de la fonction d'offre dans le cas de la valeur commune, qui est une fonction linéaire du signal, il a été possible d'obtenir les différents moments du premier et du second ordres nécessaires à l'application empirique. Toutefois, cette partie empirique a révélé les limites de l'approche en deux étapes par les moindres carrés: les valeurs du coefficient de corrélation obtenues dans la deuxième étape se sont révélées très sensibles à la valeur des coefficients estimés à la première. L'estimation directe de l'ensemble des coefficients par les moindres carrés non-linéaires a permis de confirmer l'existence d'une corrélation entre les signaux dans le cas des exportations européennes de céréales, corrélation cependant bien plus forte que celles autorisées par le type de distribution multivariée retenue. Cette limite de notre démarche structurelle n'est cependant pas la seule: il n'a pas été possible d'étendre la méthode d'estimation par les moindres carrés au cas des valeurs privées, du fait de la difficulté d'obtenir les moments théoriques du premier et du second ordre nécessaires à cette spécification. D'autres méthodes d'estimation que les moindres carrés seraient toutefois utilisables, et du fait qu'il est notamment possible de spécifier la distribution des deux premiers signaux, la méthode du maximum de vraisemblance semble adéquate. Cette dernière nécessite toutefois $\mathrm{d}$ ' "inverser" la fonction d'offre, ce qui rend la mise en oeuvre d'une telle méthode particulièrement délicate. 


\section{Annexes}

\section{A Stratégie d'équilibre sous l'hypothèse de valeurs privées}

Rappelons que la distribution conditionnelle du second signal sachant le premier s'exprime:

avec

$$
F_{Y_{2}}\left(y_{2} \mid y_{1}\right)=\frac{\partial F_{1,2}\left(y_{1}, y_{2}\right) / \partial y_{1}}{f_{1}\left(y_{1}\right)}
$$

$$
\begin{aligned}
\frac{\partial F_{1,2}\left(y_{1}, y_{2}\right)}{\partial y_{1}} & =\frac{d}{d y_{1}}\left[n\left(1+r(n-1) S\left(y_{2}\right)\left(S\left(y_{1}\right)+S\left(y_{2}\right) \frac{n-2}{2}\right)\right) F\left(y_{1}\right) F\left(y_{2}\right)^{n-1}\right] \\
& =n f\left(y_{1}\right) F\left(y_{2}\right)^{n-1}\left(1+r(n-1) S\left(y_{2}\right)\left(2 S\left(y_{1}\right)-1+\frac{n-2}{2} S\left(y_{2}\right)\right)\right)
\end{aligned}
$$

La densité associée, $f_{1,2}\left(y_{1}, y_{2}\right)$, s'écrit:

$$
\begin{aligned}
f_{1,2}\left(y_{1}, y_{2}\right)=\frac{\partial^{2} F_{1,2}\left(y_{1}, y_{2}\right)}{\partial y_{1} \partial y_{2}} & \\
= & n(n-1) f\left(y_{1}\right) F\left(y_{2}\right)^{n-2} f\left(y_{2}\right)\left(1+r(n-1) S\left(y_{2}\right)\left(2 S\left(y_{1}\right)-1+\frac{n-2}{2} S\left(y_{2}\right)\right)\right) \\
& -n f\left(y_{1}\right) F\left(y_{2}\right)^{n-1} f\left(y_{2}\right) r(n-1)\left(2 S\left(y_{1}\right)-1+(n-2) S\left(y_{2}\right)\right)
\end{aligned}
$$

Le ratio de vraisemblance s'exprime par conséquent :

$\frac{f_{Y_{2}}\left(y_{2} \mid y_{1}\right)}{F_{Y_{2}}\left(y_{2} \mid y_{1}\right)}=(n-1) \frac{f\left(y_{2}\right)}{F\left(y_{2}\right)}-f\left(y_{2}\right) \frac{r(n-1)\left(2 S\left(y_{1}\right)-1+(n-2) S\left(y_{2}\right)\right)}{1+r(n-1) S\left(y_{2}\right)\left(2 S\left(y_{1}\right)-1+\frac{n-2}{2} S\left(y_{2}\right)\right)}$

et l'on a :

$$
\frac{f_{Y_{2}}(y \mid y)}{F_{Y_{2}}(y \mid y)}=(n-1) \frac{f(y)}{F(y)}-\frac{r(n-1)(n S(y)-1) f(y)}{1+r(n-1)\left(\frac{n+2}{2} S(y)-1\right) S(y)}
$$

et d'après (2), il vient

$$
L\left(y \mid a_{i}\right)=\frac{F(y)^{n-1}}{F\left(a_{i}\right)^{n-1}} \exp \left(\int_{y}^{a_{i}} \frac{r(n-1)(n S(a)-1) f(a)}{1+r(n-1)\left(\frac{n+2}{2} S(a)-1\right) S(a)} d a\right)
$$




\section{B Terme intégral dans le cas d'une distribu- tion uniforme}

Dans le cas d'une distribution uniforme sur un intervalle $[\underline{a}, \bar{a}]$, le terme $C\left(a_{i}\right)$ s'exprime

$$
C\left(a_{i}\right)=\int_{\underline{a}}^{a_{i}} \frac{(y-\underline{a})^{n-1}}{\left(a_{i}-\underline{a}\right)^{n-1}}\left(\frac{(\bar{a}-y)+\left(\bar{a}-a_{i}\right)}{2(\bar{a}-\underline{a})}-\frac{1}{n}\right) \frac{(y-\underline{a})-\left(a_{i}-\underline{a}\right)}{\bar{a}-\underline{a}} d y
$$

ou de manière équivalente (en posant $a=y-\underline{a}$ et $x_{i}=a_{i}-\underline{a}$ ),

$$
\begin{aligned}
C\left(x_{i}+\underline{a}\right) & =\int_{0}^{x_{i}} \frac{a^{n-1}}{x_{i}^{n-1}}\left(\frac{n-1}{n}-\frac{a+x_{i}}{2(\bar{a}-\underline{a})}\right) \frac{x_{i}-a}{\bar{a}-\underline{a}} d a \\
& =\frac{1}{x_{i}^{n-1} 2 n(\bar{a}-\underline{a})^{2}} \int_{0}^{x_{i}} a^{n-1}\left(2(\bar{a}-\underline{a})(n-1)-n\left(a+x_{i}\right)\right)\left(x_{i}-a\right) d a \\
& =\frac{1}{x_{i}^{n-1} 2 n(\bar{a}-\underline{a})^{2}} \int_{0}^{x_{i}} \frac{a^{n}}{n}(2(\bar{a}-\underline{a})(n-1)-2 n a) d a \\
& =\frac{1}{x_{i}^{n-1} 2 n(\bar{a}-\underline{a})^{2}}\left(\frac{2 x_{i}^{n+1}(\bar{a}-\underline{a})(n-1)}{n(n+1)}-\frac{2 x_{i}^{n+2}}{n+2}\right) \\
& =\frac{x_{i}^{2}}{n(\bar{a}-\underline{a})^{2}}\left[\frac{(\bar{a}-\underline{a})(n-1)}{n(n+1)}-\frac{x_{i}}{n+2}\right]
\end{aligned}
$$

et $C\left(a_{i}\right)$ est négatif si

$$
\frac{a_{i}-\underline{a}}{\bar{a}-\underline{a}}>\frac{(n-1)(n+2)}{n(n+1)}
$$

\section{Stratégie d'équilibre sous l'hypothèse de valeur commune}

En remarquant que $f\left(a_{i} \mid c\right)=f\left(c \mid a_{i}\right)$, la densité marginale du couple des deux meilleurs signaux s'écrit, d'après (19),

$$
\begin{aligned}
f_{1,2}(y, y) \equiv & \int_{-\infty}^{+\infty} f_{1,2}(y, y \mid c) d c \\
= & n(n-1) \int_{-\infty}^{+\infty} F(c \mid y)^{n-2} f(c \mid y)^{2} d c \\
& +r n(n-1)^{2} \int_{-\infty}^{+\infty} F(c \mid y)^{n-2} f(c \mid y)^{2} S(c \mid y)\left(\frac{n+2}{2} S(c \mid y)-1\right) d c
\end{aligned}
$$




$$
\begin{aligned}
& -r n(n-1) \int_{-\infty}^{+\infty} F(c \mid y)^{n-1} f(c \mid y)^{2}(n S(c \mid y)-1) d c \\
= & I_{1}+r n(n-1)^{2} I_{2}-r n(n-1) I_{3}
\end{aligned}
$$

avec :

$$
\begin{aligned}
I_{1}= & n(n-1) \int_{-\infty}^{+\infty} F(c \mid y)^{n-2} f(c \mid y)^{2} d c \\
I_{2}= & \int_{-\infty}^{+\infty} F(c \mid y)^{n-2} f(c \mid y)^{2}\left(\frac{n+2}{2} S(c \mid y)^{2}-S(c \mid y)\right) d c \\
= & \int_{-\infty}^{+\infty} F(c \mid y)^{n-2} f(c \mid y)^{2}\left(\frac{n+2}{2}\left(1-2 F(c \mid y)+F(c \mid y)^{2}\right)-1+F(c \mid y)\right) d c \\
= & \frac{n}{2} \int_{-\infty}^{+\infty} F(c \mid y)^{n-2} f(c \mid y)^{2} d c-(n+1) \int_{-\infty}^{+\infty} F(c \mid y)^{n-1} f(c \mid y)^{2} d c \\
& +\frac{n+2}{2} \int_{-\infty}^{+\infty} F(c \mid y)^{n} f(c \mid y)^{2} d c \\
I_{3}= & \int_{-\infty}^{+\infty} F(c \mid y)^{n-1} f(c \mid y)^{2}(n-1-n F(c \mid y)) d c \\
= & (n-1) \int_{-\infty}^{+\infty} F(c \mid y)^{n-1} f(c \mid y)^{2} d c-n \int_{-\infty}^{+\infty} F(c \mid y)^{n} f(c \mid y)^{2} d c
\end{aligned}
$$

En remarquant que

$$
\begin{aligned}
\int_{-\infty}^{+\infty} F(c \mid y)^{k} f(c \mid y)^{2} d c & =\frac{1}{k+1} \int_{-\infty}^{+\infty} f(c \mid y) d F(c \mid y)^{k+1} \\
& =\frac{1}{k+1}\left(\left[f(c \mid y) F(c \mid y)^{k+1}\right]_{-\infty}^{+\infty}-\int_{-\infty}^{+\infty} f^{\prime}(c \mid y) F(c \mid y)^{k+1} d c\right) \\
& =-\frac{1}{k+1} \int_{-\infty}^{+\infty} f^{\prime}(c \mid y) F(c \mid y)^{k+1} d c
\end{aligned}
$$

où

$$
f^{\prime}(c \mid y)=-\left(\frac{c-y}{\sigma^{2}}\right) f(c \mid y)
$$

on obtient

$$
\begin{aligned}
\int_{-\infty}^{+\infty} F(c \mid y)^{k} f(c \mid y)^{2} d c & =\frac{1}{(k+1)(k+2) \sigma} \int_{-\infty}^{+\infty} \frac{c-y}{\sigma} d F(c \mid y)^{k+2} \\
& =\frac{1}{(k+1)(k+2) \sigma} \int_{-\infty}^{+\infty} t d \Phi(t)^{k+2} \\
& \equiv \frac{E_{k+2}[t]}{(k+1)(k+2) \sigma}
\end{aligned}
$$


Par conséquent :

$$
\begin{aligned}
& I_{1}=E_{n}[t] / \sigma \\
& I_{2}=\frac{E_{n}[t]}{2(n-1) \sigma}-\frac{E_{n+1}[t]}{n \sigma}+\frac{E_{n+2}[t]}{2(n+1) \sigma} \\
& I_{3}=\frac{(n-1) E_{n+1}[t]}{n(n+1) \sigma}-\frac{n E_{n+2}[t]}{(n+2)(n+1) \sigma}
\end{aligned}
$$

et finalement

$$
\begin{aligned}
\sigma f_{1,2}(y, y)= & E_{n}[t]+r \frac{n(n-1)}{2}\left(E_{n}[t]-\frac{2(n-1)(n+2)}{n(n+1)} E_{n+1}[t]\right. \\
& \left.+\frac{(n-1)(n+2)+2 n}{(n+1)(n+2)} E_{n+2}[t]\right) \\
= & E_{n}[t]+r \frac{n(n-1)}{2} \psi_{n}(t)
\end{aligned}
$$

La valeur estimée du bien s'écrit

$$
v(y, y)=\frac{\int_{-\infty}^{+\infty} c f_{1,2}(y, y \mid c) d c}{f_{1,2}(y, y)}
$$

avec

$$
\int_{-\infty}^{+\infty} c f_{1,2}(y, y \mid c) d c=I_{1}^{\prime}+r n(n-1)^{2} I_{2}^{\prime}-r n(n-1) I_{3}^{\prime}
$$

où

$$
\begin{aligned}
I_{1}^{\prime}= & n(n-1) \int_{-\infty}^{+\infty} c F(c \mid y)^{n-2} f(c \mid y)^{2} d c \\
I_{2}^{\prime}= & \frac{n}{2} \int_{-\infty}^{+\infty} c F(c \mid y)^{n-2} f(c \mid y)^{2} d c-(n+1) \int_{-\infty}^{+\infty} c F(c \mid y)^{n-1} f(c \mid y)^{2} d c \\
& +\frac{n+2}{2} \int_{-\infty}^{+\infty} c F(c \mid y)^{n} f(c \mid y)^{2} d c \\
I_{3}^{\prime}= & (n-1) \int_{-\infty}^{+\infty} c F(c \mid y)^{n-1} f(c \mid y)^{2} d c-n \int_{-\infty}^{+\infty} c F(c \mid y)^{n} f(c \mid y)^{2} d c
\end{aligned}
$$

En remarquant que

$$
\begin{aligned}
& \int_{-\infty}^{+\infty} c F(c \mid y)^{k} f(c \mid y)^{2} d c=-\frac{1}{k+1} \int_{-\infty}^{+\infty}\left(f(c \mid y)+c f^{\prime}(c \mid y)\right)[F(c \mid y)]^{k+1} d c \\
& =\frac{1}{(k+1)(k+2)}\left(-1+\int_{-\infty}^{+\infty}\left(\frac{c-y}{\sigma}\right)^{2} d F(c \mid y)^{k+2}+\frac{y}{\sigma} \int_{-\infty}^{+\infty} \frac{c-y}{\sigma} d F(c \mid y)^{k+2}\right)
\end{aligned}
$$




$$
\begin{aligned}
& =\frac{1}{(k+1)(k+2)}\left(-1+\int_{-\infty}^{+\infty} t^{2} d \Phi(t)^{k+2}+\frac{y}{\sigma} \int_{-\infty}^{+\infty} t d \Phi(t)^{k+2}\right) \\
& =\frac{E_{k+2}\left[t^{2}-1\right]+\frac{y}{\sigma} E_{k+2}[t]}{(k+1)(k+2)}
\end{aligned}
$$

on obtient

$$
\begin{aligned}
I_{1}^{\prime}= & E_{n}\left[t^{2}-1\right]+\frac{y}{\sigma} E_{n}[t] \\
I_{2}^{\prime}= & \frac{1}{2(n-1)}\left(E_{n}\left[t^{2}-1\right]+\frac{y}{\sigma} E_{n}[t]\right)-\frac{1}{n}\left(E_{n+1}\left[t^{2}-1\right]+\frac{y}{\sigma} E_{n+1}[t]\right) \\
& +\frac{1}{2(n+1)}\left(E_{n+2}\left[t^{2}-1\right]+\frac{y}{\sigma} E_{n+2}[t]\right) \\
I_{3}^{\prime}= & \frac{n-1}{n(n+1)}\left(E_{n+1}\left[t^{2}-1\right]+\frac{y}{\sigma} E_{n+1}[t]\right) \\
& -\frac{n}{(n+1)(n+2)}\left(E_{n+2}\left[t^{2}-1\right]+\frac{y}{\sigma} E_{n+2}[t]\right)
\end{aligned}
$$

et par conséquent:

$$
\begin{aligned}
\int_{-\infty}^{+\infty} c f_{1,2}(y, y \mid c) d c= & (1+r n(n-1) / 2)\left(E_{n}\left[t^{2}-1\right]+\frac{y}{\sigma} E_{n}[t]\right) \\
& -r(n-1)^{2} \frac{2 n+1}{n+1}\left(E_{n+1}\left[t^{2}-1\right]+\frac{y}{\sigma} E_{n+1}[t]\right) \\
& +r \frac{n(n-1)}{(n+1)}\left(\frac{n-1}{2}+\frac{n}{n+2}\right)\left(E_{n+2}\left[t^{2}-1\right]+\frac{y}{\sigma} E_{n+2}[t]\right) \\
= & E_{n}\left[t^{2}-1\right]+r \frac{n(n-1)}{2} \psi\left(t^{2}-1\right)+\frac{y}{\sigma}\left(E_{n}[t]+r \frac{n(n-1)}{2} \psi(t)\right)
\end{aligned}
$$

ce qui donne finalement

$$
v(y, y)=y+\sigma \frac{E_{n}\left[t^{2}-1\right]+r \frac{n(n-1)}{2} \psi\left(t^{2}-1\right)}{E_{n}[t]+r \frac{n(n-1)}{2} \psi(t)}
$$

qui est linéaire en $y$. Enfin, observons que

$$
\begin{aligned}
& \frac{\partial F_{1,2}(y, y)}{\partial y_{1}} \equiv \int_{-\infty}^{+\infty} \frac{\partial F_{1,2}(y, y \mid c)}{\partial y_{1}} d c \\
& =\int_{-\infty}^{+\infty} n f(c \mid y) F(c \mid y)^{n-1}\left(1+r(n-1) S(c \mid y)\left(\frac{n+2}{2} S(c \mid y)-1\right)\right) d c \\
& =1+r n(n-1) \int_{-\infty}^{+\infty} n f(c \mid y) F(c \mid y)^{n-1}\left(\frac{n+2}{2}\left(1-2 F(c \mid y)+F(c \mid y)^{2}\right)-1+F(c \mid y)\right) d c
\end{aligned}
$$




$$
\begin{aligned}
= & 1+r n(n-1) \int_{-\infty}^{+\infty}\left(\frac{n}{2} f(c \mid y) F(c \mid y)^{n-1}\right. \\
& \left.-(n+1) f(c \mid y) F(c \mid y)^{n}+\frac{n+2}{2} f(c \mid y) F(c \mid y)^{n+1}\right) d c \\
= & 1+r n(n-1)\left(\frac{1}{2}-1+\frac{1}{2}\right) \\
= & 1
\end{aligned}
$$

de sorte que :

$$
L\left(y \mid a_{i}\right)=\exp \left(\left(y-a_{i}\right) f_{1,2}\right)
$$

et, comme $f_{1,2}$ est une constante,

$$
\begin{aligned}
\int_{\underline{a}}^{a_{i}} L\left(y \mid a_{i}\right) d v(y, y) & =\frac{1}{f_{1,2}}\left[\exp \left(\left(y-a_{i}\right) f_{1,2}\right)\right]_{-\infty}^{a_{i}} \\
& =\frac{1}{f_{1,2}}
\end{aligned}
$$

La stratégie d'équilibre s'exprime finalement

$$
\begin{aligned}
b^{*}\left(a_{i}\right) & =v\left(a_{i}, a_{i}\right)-\int_{\underline{a}}^{a_{i}} L\left(y \mid a_{i}\right) d v(y, y) \\
& =a_{i}-\sigma \frac{E_{n}\left[t^{2}\right]+r n(n-1) \psi\left(t^{2}-1\right) / 2}{E_{n}[t]+r n(n-1) \psi(t) / 2}
\end{aligned}
$$

\section{Espérances et des variances des deux plus grandes offres}

L'offre gagnante s'exprime:

$$
b^{w}=a^{w}-\sigma \alpha_{n}(r)
$$

où $a^{w}$ est le signal du vainqueur, dont l'espérance s'exprime (d'après (6)) :

$$
\begin{aligned}
& E\left[A^{w} \mid C\right]=\int_{-\infty}^{+\infty} y n F(y \mid c)^{n-1} f(y \mid c)\left(1+\frac{n-1}{2} r S(y \mid c)((n+2) S(y \mid c)-2)\right) d y \\
& =\int_{-\infty}^{+\infty} y d F(y \mid c)^{n} \\
& \quad+r \frac{n(n-1)}{2} \int_{-\infty}^{+\infty} y F(y \mid c)^{n-1} f(y \mid c)\left((n+2) F(y \mid c)^{2}-2(n+1) F(y \mid c)+n\right) d y
\end{aligned}
$$




$$
\begin{aligned}
= & \int_{-\infty}^{+\infty} y d F(y \mid c)^{n} \\
& +r \frac{n(n-1)}{2}\left(\int_{-\infty}^{+\infty} y d F(y \mid c)^{n+2}-2 \int_{-\infty}^{+\infty} y d F(y \mid c)^{n+1}+\int_{-\infty}^{+\infty} y d F(y \mid c)^{n}\right)
\end{aligned}
$$

où $\int_{-\infty}^{+\infty} y d F(y \mid c)^{k}=\int_{-\infty}^{+\infty}(\sigma t+c) d \Phi(t)^{k}=c+\sigma E_{k}[t]$, de sorte que:

$$
\begin{aligned}
E\left[A^{w} \mid C\right] & =c+\sigma\left(E_{n}[t]+r \frac{n(n-1)}{2}\left(E_{n+2}[t]-2 E_{n+1}[t]+E_{n}[t]\right)\right) \\
& \equiv c+\sigma \varphi_{1}(r, t)
\end{aligned}
$$

La variance de l'offre gagnante s'exprime:

$$
\begin{aligned}
V\left[B^{w} \mid C\right] & =E\left[\left(B^{w}-E\left[B^{w}\right]\right)^{2} \mid C\right] \\
& =E\left[\left(A^{w}-c-\sigma \varphi_{1}(t)\right)^{2} \mid C\right] \\
& =E\left[\left(A^{w}-c\right)^{2} \mid C\right]+\sigma^{2} \varphi_{1}(t, r)^{2}-2 \sigma \varphi_{1}(t)\left(E\left[A^{w} \mid C\right]-c\right) \\
& =E\left[\left(A^{w}-c\right)^{2} \mid C\right]-\sigma^{2} \varphi_{1}(t, r)^{2}
\end{aligned}
$$

où :

$$
\begin{aligned}
E\left[\left(A^{w}-c\right)^{2} \mid C\right] & =\int_{-\infty}^{+\infty}(y-c)^{2} d F_{1}(c \mid y) \\
& =\sigma^{2} \int_{-\infty}^{+\infty}\left(\frac{y-c}{\sigma}\right)^{2} d F_{1}(c \mid y) \\
& =\sigma^{2} \int_{-\infty}^{+\infty} t^{2} d \Phi_{1}(t) \\
& =\sigma^{2} \varphi_{1}\left(t^{2}, r\right)
\end{aligned}
$$

Par conséquent:

$$
V\left[B^{w} \mid C\right]=\sigma^{2}\left(\varphi_{1}\left(t^{2}, r\right)-\varphi_{1}(t, r)^{2}\right)
$$

Nous avons:

$$
\begin{aligned}
F_{2}(x) & =P\left[\bigcup_{i}\left(\bigcap_{j \neq i}\left(V_{j} \leq x\right) \bigcap\left(V_{i}>x\right)\right)\right]+P\left[\bigcap_{i}\left(V_{i} \leq x\right)\right] \\
& =n F(x)^{n-1} S(x)\left(1+r(n-1) F(x) S(x)+r \frac{(n-1)(n-2)}{2} S(x)^{2}\right)+F_{1}(x) \\
& =F_{(2 \mid n)}(x)+r \frac{n(n-1)}{2} F(x)^{n-1} S(x)^{2}(2 F(x)+(n-2) S(x)+F(x)) \\
& =F_{(2 \mid n)}(x)+r \frac{n(n-1)}{2} F(x)^{n-1} S(x)^{2}((n-5) S(x)+3)
\end{aligned}
$$


avec

$$
\begin{aligned}
S(x)^{2}((n-5) S(x)+3) & =(n-5) S(x)^{3}+3 S(x)^{2} \\
& =(n-5)\left(1-3 F(x)+3 F(x)^{2}-F(x)^{3}\right)+3\left(1-2 F(x)+F(x)^{2}\right)
\end{aligned}
$$

Par conséquent,

$$
\begin{aligned}
F_{2}(x)= & F_{(2 \mid n)}(x)+r \frac{n(n-1)}{2} \times \\
& \left((n-2) F(x)^{n-1}-3(n-3) F(x)^{n}+3(n-4) F(x)^{n+1}-(n-5) F(x)^{n+2}\right) .
\end{aligned}
$$

et l'espérance du deuxième signal s'écrit:

$$
\begin{aligned}
E\left[A_{(2)}\right] & =\varphi_{2}(a, r) \\
& =c+\sigma \varphi_{2}(t, r)
\end{aligned}
$$

La variance de la deuxième offre s'exprime:

$$
\begin{aligned}
V\left[B_{(2)} \mid C\right] & =E\left[\left(B_{(2)}-E\left[B_{(2)}\right]\right)^{2}\right] \\
& =E\left[\left(A_{(2)}-c-\sigma \varphi_{2}(t, r)\right)^{2}\right] \\
& =E\left[\left(A_{(2)}-c\right)^{2}\right]+\sigma^{2} \varphi_{2}(t, r)^{2}-2 \sigma \varphi_{2}(t, r)\left(E\left[A_{(2)}\right]-c\right) \\
& =E\left[\left(A_{(2)}-c\right)^{2}\right]-\sigma^{2} \varphi_{2}(t)^{2}
\end{aligned}
$$

où :

$$
\begin{aligned}
E\left[\left(A_{(2)}-c\right)^{2}\right] & =\int_{-\infty}^{+\infty}(y-c)^{2} d F_{2}(c \mid y) \\
& =\sigma^{2} \int_{-\infty}^{+\infty}\left(\frac{y-c}{\sigma}\right)^{2} d F_{2}(c \mid y) \\
& =\sigma^{2} \varphi_{2}\left(t^{2}, r\right)
\end{aligned}
$$

ce qui donne le résultat.

\section{E Moments non centrés du second ordre}

On a :

$$
\begin{aligned}
E\left[\left(B^{w}\right)^{2} \mid C\right] & =E\left[\left(A^{w}-\sigma \alpha_{n}(r)\right)^{2} \mid C\right] \\
& =E\left[\left(A^{w}\right)^{2} \mid C\right]+\sigma^{2} \alpha_{n}(r)^{2}-2 \sigma \alpha_{n}(r) E\left[A^{w} \mid C\right]
\end{aligned}
$$


où

$$
E\left[A^{w} \mid C\right]=c+\sigma \varphi_{1}(r, t)
$$

et

$$
\begin{aligned}
E\left[\left(A^{w}\right)^{2} \mid C\right] & =E\left[\left(A^{w}-c+c\right)^{2} \mid C\right] \\
& =E\left[\left(A^{w}-c\right)^{2}+2 c\left(A^{w}-c\right)+c^{2} \mid C\right] \\
& =\sigma^{2} \varphi_{1}\left(t^{2}, r\right)+2 c E\left[A^{w}-c\right]+c^{2} \\
& =\sigma^{2} \varphi_{1}\left(t^{2}, r\right)+2 c \sigma \varphi_{1}(r, t)+c^{2}
\end{aligned}
$$

Par conséquent

$$
\begin{aligned}
E\left[\left(B^{w}\right)^{2} \mid C\right] & =\sigma^{2} \varphi_{1}\left(t^{2}, r\right)+2 c \sigma \varphi_{1}(r, t)+c^{2}+\sigma^{2} \alpha_{n}(r)^{2}-2 \sigma \alpha_{n}(r)\left(c+\sigma \varphi_{1}(r, t)\right) \\
& =\sigma^{2}\left(\varphi_{1}\left(t^{2}, r\right)+\alpha_{n}(r)^{2}-2 \alpha_{n}(r) \varphi_{1}(t, r)\right)+2 \sigma c\left(\varphi_{1}(r, t)-\alpha_{n}(r)\right)+c^{2} \\
& =\left(\sigma\left(\varphi_{1}(r, t)-\alpha_{n}(r)\right)+c\right)^{2}+\sigma^{2}\left(\varphi_{1}\left(t^{2}, r\right)-\varphi_{1}(t, r)\right)
\end{aligned}
$$

On obtient de la même manière

$$
E\left[B_{(2)}^{2} \mid C\right]=\left(\sigma\left(\varphi_{2}(r, t)-\alpha_{n}(r)\right)+c\right)^{2}+\sigma^{2}\left(\varphi_{2}\left(t^{2}, r\right)-\varphi_{2}(t, r)\right)
$$

\section{Références}

[1] R. Aumann and S. Hart, editors. Handbook of Game Theory, volume 1. Elsevier Science Publishers, 1992.

[2] J.-M. Bourgeon and Y. Le Roux. Politique europénne de céréales et comportements des opérateurs: une analyse économétrique des adjudications de restitutions. Économie et Prévision, 1/2(117-118): 31-48, 1995.

[3] C. Gouriéroux and F. Jouneau. Multivariate distributions for limited dependent variable models. Working Paper 9414, CREST, 1994.

[4] C. Gouriéroux, A. Monfort, and A. Trognon. Moindres carrés asymptotiques. Annales de l'INSEE, 58: 91-122, 1985.

[5] L. P. Hansen. Large sample properties of generalized method of moments estimators. Econometrica, 50: 1029-1054, 1982.

[6] J.-J. Laffont and Q. Vuong. Structural econometric models of strategic behavior. American Economic Review, 86(2): 414-420, 1996. 
[7] D. Levin and J. Smith. Some evidence on the winner's curse, a comment. American Economic Review, 81(1): 370-375, 1991.

[8] P. R. Milgrom and R. J. Weber. A theory of auctions and competitive bidding. Econometrica, 50(5): 1089-1122, 1982.

[9] H. J. Paarsch. Deciding between the common and private value paradigms in empirical models of auctions. Journal of Econometrics, 51(1-2): 191-215, 1992.

[10] S. E. Thiel. Some evidence on the winner's curse. American Economic Review, 78(5): 884-895, 1988.

[11] R. Wilson. Strategic Analysis of Auctions, pages 227-279. Volume 1 of Aumann and Hart [1], 1992. 